\title{
Finite-Size Scaling and Surface Tension from Effective One Dimensional Systems
}

\author{
Christian Borgs ${ }^{1, \star}$ and John Z. Imbrie ${ }^{2, \star \star}$ \\ ${ }^{1}$ Institut für Theoretische Physik, Freie Universität Berlin, Arnimallee 14, 1000 Berlin 33, \\ Federal Republic of Germany \\ 2 Departments of Mathematics and Physics, Harvard University, Cambridge, MA 02138, USA
}

Received May 17, 1991

\begin{abstract}
We develop a method for precise asymptotic analysis of partition functions near first-order phase transitions. Working in a $(v+1)$-dimensional cylinder of volume $L \times \cdots \times L \times t$, we show that leading exponentials in $t$ can be determined from a simple matrix calculation provided $t \geqq v \log L$. Through a careful surface analysis we relate the off-diagonal matrix elements of this matrix to the surface tension and $L$, while the diagonal matrix elements of this matrix are related to the metastable free energies of the model. For the off-diagonal matrix elements, which are related to the crossover length from hypercubic $(L=t)$ to cylindrical $(t=\infty)$ scaling, this includes a determination of the pre-exponential power of $L$ as a function of dimension. The results are applied to supersymmetric field theory and, in a forthcoming paper, to the finite-size scaling of the magnetization and inner energy at field and temperature driven first-order transitions in the crossover region from hypercubic to cylindrical scaling.
\end{abstract}

\section{Introduction}

Finite-size effects at first-order phase transitions exhibit a rich set of phenomena that have been brought to light by a number of authors [FB, P, PF]. Recent rigorous studies have clarified some of the issues involved with the rounding of the magnetization jump in a finite periodic hypercube with periodic boundary conditions [BK, BKM]. One may analyze the asymptotics of the partition function or the magnetization as the dimension, $\mathscr{L}$, of the hypercube tends to infinity. For example, varying the magnetic field $\mu$ of the Ising model in a neighborhood of

\footnotetext{
* Research partially supported by the A. P. Sloan Foundation and by the NSF under DMS-8858073

$\star \star$ Research partially supported by the NSF under DMS-8858073 and DMS-9008827
} 
zero, the magnetization approximates a hyperbolic tangent with transition region of width $L^{-d}$ in dimension $d>1$. The shape function is in fact universal for any two-phase system. This is due to the fact that the partition function is essentially a sum of contributions $\exp \left(\mu M_{ \pm} L^{d}\right)$ from the two phases, where $M_{ \pm}$are the infinite volume magnetizations of the plus and the minus phase, respectively (for the Ising model, $M_{-}=-M_{+}$). The logarithmic derivative of the sum produces the characteristic tanh shape.

In this paper we consider systems in cylindrical $(v+1)$-dimensional periodic boxes having dimensions $L \times \cdots \times L \times t$. A new set of phenomena will come to light as we investigate the joint asymptotics as $L, t$ tend to infinity (under the limitation $\left.L^{v} e^{-t} \leqq 1\right)$. Such geometries are of interest when studying the lowest eigenvalues of a quantum mechanical Hamiltonian. (A v-dimensional quantum system can frequently be rewritten as $(v+1)$-dimensional classical statistical mechanics, with $t=\beta$, the inverse temperature.) It is particularly interesting to see how the shape function crosses over from the hyperbolic tangent (found when $L=t \rightarrow \infty$ ) to the shape characteristic of one-dimensional systems (expected when $t \rightarrow \infty$ before $L \rightarrow \infty$ ). The latter shape may be found by computing the largest eigenvalue of the transfer matrix of the one-dimensional Ising model,

$$
T=\left(\begin{array}{cc}
e^{\mu} & e^{-\beta} \\
e^{-\beta} & e^{-\mu}
\end{array}\right),
$$

and taking its logarithmic $\mu$-derivative near $\mu=0$.

For systems describing the coexistence of two phases related by a symmetry, Privman and Fischer developed a heuristic theory of crossover finite-size scaling which predicts a crossover from hypercubic to cylindrical scaling when $t$ diverges with $L$ like $\xi_{L}$, where

$$
\xi_{L} \sim \text { const } L^{w} \exp \left(\sigma L^{v}\right)
$$

is the inverse mass gap at the transition point, $\sigma$ is the surface tension between the two phases and $w$ is a dimension dependent exponent. Privman and Fisher [PF] predicted $w=1 / 2$ for $v=1$ but gave no prediction for $v>1$. Brezin and Zinn-Justin [BZ] predicted $w=(2-v) / 2$ (see also [BCJZ]).

The theory presented here starts from a detailed analysis of the microscopic configurations of the system. It turns out that the most important effects of the cylinder geometry are related to the spontaneous formation of interfaces, separating two different phases above and below the interface. Rewriting the periodic partition function as a weakly interacting gas of such interfaces, and using renormalization group ideas to control this interaction, we will be able to reduce the partition function to the partition function of an effective one dimensional spin system. As a result, we are able to calculate the partition function asymptotics of a system describing the coexistence of $N$ phases (not necessarily related by a symmetry) in terms of a $N \times N$ symmetric matrix.

Our results conform, in the Ising case, with the picture developed by Privman and Fisher $[\mathrm{PF}]$ for crossover finite-size scaling. Our aim is to put crossover scaling on a rigorous footing, to treat a wide class of $N$-phase systems, and to give concrete estimates on the deviations from ideal scaling behavior. In addition, we determine the exponent $w=w(v)$ in the relation (1.2). We will show that at low 
temperature,

$$
w=\left\{\begin{array}{lll}
1 / 2 & \text { if } & v+1=2 \\
0 & \text { if } & v+1>2
\end{array}\right.
$$

confirming thus the prediction of Privman and Fisher for $v+1=2$, and correcting the prediction of Brezin and Zinn-Justin for $v+1>3$. We also determine that the constant is $1 / 2$ at low temperature if $v+1>2$; it is not universal in $v+1=2$ dimensions.

Main Results. We now state two theorems, one relating the partition function with periodic boundary conditions to the partition function of an effective one-dimensional model, and one relating the off-diagonal matrix elements of the corresponding transfer matrix to the string tension of the original model and to $L$. We defer to Sect. 2 a description of the class of models to which the theorems apply. Essentially we need a contour or cluster representation with a Peierls condition, translation invariance, and invariance under reflection in the $t$-direction. Thus our results apply to perturbed Ising models at low temperature, to large $q$ Potts models [LMMRS], to large $N$ lattice Higgs models [BFW] and to continuous spin systems and quantum field models near first-order transitions [I, JW1]. The notation is as follows: $Z_{\text {per }}(V)$ is the periodic partition function in volume $V,|V|=L^{v} t, \mu$ is an $(N-1)$-vector of parameters driving transitions amongst $N$ states, $\mu^{*}$ is the coexistence point, $f(\mu)$ is the free energy density, and $\tau$ is the parameter in the Peierls condition ( $\tau=\beta$ for the Ising model). It is assumed throughout that $L, t$ are positive integers.

Theorem A. There are $C^{4}$ functions $f_{q}(\mu) \geqq f(\mu), q=1, \ldots, N$, agreeing with $f(\mu)$ is and only if the corresponding phase is stable, such that the following statements are true provided $\tau$ is sufficiently large and $\left|\mu-\mu^{*}\right| L^{\nu} \leqq 1$.

(i) There exists an $N \times N$ symmetric matrix $M$ such that for all $t \geqq v \log L$ and for $0 \leqq k \leqq 4$,

$$
\begin{gathered}
\left|\frac{d^{k}}{d \mu^{k}}\left(Z_{\mathrm{per}}(V)-\operatorname{Tr} M^{t}\right)\right| \leqq e^{-f|V|} e^{-O(\tau) t}, \\
\left|\frac{d^{k}}{d \mu^{k}}\left(L^{-v} \log M_{q q}+f_{q}(\mu)\right)\right| \leqq e^{-(\tau-O(1)) L}, \\
\left|\frac{d^{k}}{d \mu^{k}} M_{p q}(L)\right| \leqq e^{-(f+\tau-O(1)) L^{v}} \quad \text { if } \quad p \neq q .
\end{gathered}
$$

This theorem reduces the determination of the asymptotics of $\mathrm{Z}_{\text {per }}(V)$ to a calculation of the eigenvalues of an $N \times N$ matrix $M$. For $N=2$, this matrix resembles (1.1) with $\pm \mu \rightarrow\left(\mu-\mu^{*}\right) L^{\nu} \frac{\partial f}{\partial \mu}\left(\mu^{* \pm}\right)$ and $e^{-\beta}$ replaced with something of the order $\exp \left(-O(\tau) L^{v}\right)$. If the original model has a positive transfer matrix $T$, as many models of statistical mechanics do,

$$
Z_{\mathrm{per}}(V)=\sum_{i=1}^{\infty} \lambda_{i}^{t}
$$


where $\lambda_{1} \geqq \lambda_{2} \geqq \cdots$ are the eigenvalues of $T$. Theorem A then implies that the first $N$ eigenvalues of $T$ are just the eigenvalues of $M$, and that $\lambda_{i} \leqq e^{-O(\tau)} \lambda_{1}$ for the remaining ones. For these models, a bound of the form (1.4) therefore remains valid for $t<v \log L$ as well.

The next theorem relates the off-diagonal matrix elements $M_{p q}$ of $M$ to the string tensions $\sigma_{p q}$ of the original model. We make several assumptions on the structure of interfaces, see Sect. 5. Essentially we need locality and suppression of defects relative to a flat interface. This makes the surface tensions $\sigma_{p q}$ well defined and enables us to connect them with the off-diagonal matrix elements of $M$.

Theorem B. Let $\tau$ be sufficiently large, $p \neq q$, and let Assumptions 5.1 through 5.5 of Sect. 5 be satisfied. Then there are constants $0<b_{1}<1$ and $C_{p q}>0$ such that

$$
\begin{array}{ll}
M_{p q}(L)=\left(1+O\left(e^{-b_{1} \tau L}\right)\right) e^{-\sigma_{p q} L^{v}} e^{-f(\mu) L^{\nu}}, & v \geqq 2, \\
M_{p q}(L)=\left(1+O\left(L^{-1}\right)\right) C_{p q} L^{-1 / 2} e^{-\sigma_{p q} L^{v}} e^{-f(\mu) L^{v}}, & v=1, \quad L \gg 1,
\end{array}
$$

provided $\left|\mu-\mu^{*}\right| \leqq e^{-\tau L / 2}$.

We will see in a subsequent paper [BI2] that the crossover length $\xi_{L}$ for $N=2$ is the inverse eigenvalue splitting at the point $\mu^{*}(L)$, where $\Gamma_{--}=\Gamma_{++}$. Calculating the eigenvalues of the $2 \times 2$ matrix $M$ and using the fact that $\left|\mu^{*}(L)-\mu^{*}\right| \leqq e^{-(\tau-o(1)) L}$ due to Theorem A, the bounds (1.4) through (1.7) immediately imply that $\xi_{L}$ is given by (1.2) and (1.3).

Using our methods, an analog to Theorem A could be proven for models with complex parameters or for models lacking reflection invariance. But then $M$ would no longer be real and symmetric, and any subsequent analysis would necessarily diverge from the treatment given here. Some interesting new phenomena can arise (for example, $Z_{\mathrm{per}}(V)$ may have zeros as $\mu$ is varied) but we do not pursue such models here.

Application to supersymmetric models. In [IJW, JW1] it was shown that the weakly coupled two-dimensional two-component Wess-Zumino models admit a contour expansion of the type required for Theorem A. In fact if we consider antiperiodic instead of periodic boundary conditions in the $t$-direction, Theorem A remains valid with the same $M$ and $f$. This provides an opportunity of proving a vanishing theorem. Let $n_{+}\left(n_{-}\right)$be the number of bosonic (fermionic) zero-energy states for the quantum mechanical Hamiltonian at fixed $t$ and finite $L$. The index theorem of [JLW] states that

$$
Z_{\text {per }}(V)=n_{+}-n_{-}=\operatorname{deg} W-1,
$$

where $W$ is the superpotential. We now prove that for these models $n_{+}+n_{-}=n_{+}-n_{-}$, in other words $n_{-}=0$ (vanishing theorem) and $n_{+}=\operatorname{deg} W-1$. (A direct proof is also possible [JW2].)

The argument proceeds as follows. It was shown already in [JW1] that $f=0$ and that $\mu=\mu^{*}$ (i.e. each of the $\operatorname{deg} W-1$ minima of the bosonic potential leads to a distinct phase in the thermodynamic limit). Hence the condition $\left|\mu-\mu^{*}\right| L^{\nu} \leqq 1$ holds for all $L$ and we have $N=\operatorname{deg} W-1$. Applying Theorem A to the periodic partition function $Z_{\text {per }}(V)=N$ and letting $t \rightarrow \infty$ we have that

$$
\lim _{t \rightarrow \infty}\left|N-\operatorname{Tr} M^{t}\right|=0 .
$$


This is possible only if $M$ is the identity matrix. The ordinary Feynman-Kac formula and $H \geqq 0$ imply that

$$
n_{+}+n_{-}=\lim _{\beta \rightarrow \infty} \operatorname{Tr} e^{-\beta H}=\lim _{t \rightarrow \infty} Z_{\mathrm{AP}}(V),
$$

where $Z_{\mathrm{AP}}(V)$ is the partition function with antiperiodic boundary conditions. Since Theorem $\mathrm{A}$ applies to $Z_{\mathrm{AP}}(V)$ with the same $M$, we see that

$$
n_{+}+n_{-}=\lim _{t \rightarrow \infty} \operatorname{Tr} M^{t}=N
$$

and the vanishing theorem is proven.

Actually $f=0$ and $\mu=\mu^{*}$ follow from the asymptotics of this paper as well. One needs to use Theorem 2.2 below, which admits for the possibility that $\left|\mu-\mu^{*}\right|>L^{-\nu}$. Then one gets estimates similar to (1.4), only the matrix $M$ may be of lower rank. Nevertheless (1.8) is incompatible with any possibility except $f=0, M=I, \operatorname{rank} M=N=\operatorname{deg} W-1$.

Outline. After definitions and precise statements of results in the next section, we develop the partition function asymptotics in Sects. 3 and 4. Section 5 is devoted to a detailed surface analysis relating $M$ to the surface tension in a neighborhood of $\mu^{*}$. Section 2 also contains a brief summary of the ideas used in the proof of Theorem $\mathbf{A}$ and Theorem $\mathbf{B}$.

\section{Definitions and Main Results}

We consider models defined in a $(v+1)$-dimensional cylinder $V=A \times T$, where $A$ is a $v$-dimensional torus with sides of length $L$ in each direction, and $T$ is a one-dimensional torus of length $t$. We assume that the model allows for an expansion into excitations $Y$ of $N$ different ground states $q \in Q=\{1, \ldots, N\}$, with real ground state energies $e_{q}$ and small activities $\rho(Y) \in \mathbb{R}$ for the excitations (henceforth called contours.) To make this precise we make the following definitions.

A contour is a pair $(Y, q(\cdot))$ where $Y$ is a connected union of unit cubes $c$ with centers in $\mathbb{Z}^{\nu+1}$ and $(q(\cdot))$ is an assignment of labels $q(F) \in Q$ to the boundaries $F$ of the components $C$ of $Y^{c}=V \backslash Y$. $Y$ is connected using shared $v$-dimensional faces as a criterion for connecting two cubes of $Y$. As a subset of $V, Y$ is considered as an open set obtained by taking the closed union of cubes in $Y$, and deleting the boundary of the resulting set. To simplify formulae, we use the symbol $Y$ to denote the pair $(Y, q(\cdot))$ as well as the region $Y$. To each contour $Y$ we assign an activity $\rho(Y) \in \mathbb{R}$ which is translation-invariant and invariant under reflections with respect to the planes $A \times t_{0}$ and satisfies the following bound for some large $\tau$ :

$$
|\rho(Y)| \leqq e^{-\left(\tau+e_{0}\right)|Y|}
$$

Here $|Y|$ denotes the volume of $Y$ and $e_{0}$ is defined as the energy of the lowest ground state,

$$
e_{0}=\min _{q} e_{q}
$$


An allowed configuration of our system is a collection, $\left\{Y_{\alpha}\right\}$, of nonoverlapping (i.e. sharing no common cubes) contours with compatible boundary labels. The compatibility is determined by the requirement that any connected component of $V \backslash \bigcup_{\alpha} Y_{\alpha}$ has constant boundary conditions. Given a collection of contours, we finally attach energy densities to the regions occupied by each phase of the model. A connected component of $V \backslash \bigcup_{\alpha} Y_{\alpha}$ that has boundary condition $m$ is considered to be part of $R_{m}$, the region "in the $m$-th phase." Thus we have partitioned $V \backslash \bigcup_{\alpha} Y_{\alpha}$ as $\bigcup_{m} R_{m}$. Note that the collection $\left\{Y_{\alpha}\right\}$ uniquely determines the regions $R_{m}$, except for the case where $\left\{Y_{\alpha}\right\}$ is empty, which corresponds to the $N$ different cases $\left|R_{m}\right|=|V| \delta_{m q}, q=1, \ldots, N$. Associating the energy density $e_{m}$ with the region $R_{m}$, we get the expression for the partition function:

$$
Z_{\text {per }}(V)=\sum_{\left\{Y_{\alpha}\right\}} \prod_{\alpha} \rho\left(Y_{\alpha}\right) \prod_{m=1}^{N} e^{-e_{m}\left|R_{m}\right|} .
$$

The connection between this partition function and the Peierls contour picture of spin systems is clear - we have just replaced sites with cubes and thickened contours to include neighboring cubes.

Next we introduce additional real parameters $\left\{\mu_{i}\right\}$ on which the activities $\rho$ and the energies $e_{q}$ may depend. There should be at least $N-1$ such parameters, and we need a degeneracy-breaking condition. Namely, we suppose that the matrix

$$
E=\left(\frac{\partial}{\partial \mu_{i}}\left(e_{q}-e_{N}\right)\right)_{q, i=1, \ldots, N-1}
$$

is nonsingular. We further assume that $\rho$ and $e_{q}$ are $C^{4}$ functions of $\mu=\left(\mu_{1}, \ldots, \mu_{N-1}\right)$ satisfying the bounds

$$
\begin{aligned}
\left|\frac{d^{k} e_{q}}{d \mu^{k}}\right| & \leqq C_{k}, \\
\left|\frac{d^{k} \rho(Y)}{d \mu^{k}}\right| & \leqq C_{k} e^{-\left(\tau+e_{0}\right)|Y|}, \quad \text { and } \\
\left\|E^{-1}\right\|_{\infty} & \equiv \max _{i} \sum_{q}\left|\left(E^{-1}\right)_{i q}\right| \leqq \text { const }<\infty,
\end{aligned}
$$

where the constants are independent of $\tau$ and $k:\{1, \ldots, N-1\} \rightarrow\{0,1, \ldots\}$ is a multiindex of order $|k| \equiv \sum k_{i}$ between $^{1} 1$ and 4 . We also assume that

$$
e_{q}\left(\mu=\mu_{0}\right)=e_{\tilde{q}}\left(\mu=\mu_{0}\right) \text { for all } q, \tilde{q} \in Q
$$

and some $\mu_{0} \in \mathbb{R}^{n-1}$.

Before stating the results proven in this paper we summarize the main facts which are known about the finite-size behavior in cubic or nearly cubic volumes $V$

\footnotetext{
${ }^{1}$ Following [BK] we take derivatives up to $4^{\text {th }}$ order. There it was used to evaluate the location of the maximum of the susceptibility, see [BK], Sect. 4
} 
(see condition (2.11) below). We introduce the free energy

$$
f(\mu)=-\lim _{V \rightarrow \mathbb{Z}^{v+1}} \frac{1}{|V|} \log Z_{\text {per }}(V, \mu)
$$

and the finite-volume order parameter

$$
M_{\mathrm{per}}^{i}(V, \mu)=\frac{1}{|V|} \frac{d}{d \mu_{i}} \log Z_{\mathrm{per}}(V, \mu),
$$

where we changed our notation with respect to $(2.3)$ to make the dependence of $Z_{\text {per }}(V)$ on $\mu$ explicit. The main result of [BK] can then be summarized by the following theorem (the construction of the functions $f_{q}$ mentioned in the theorem is sketched in Appendix A).

Theorem 2.1. There exist four times differentiable, real-valued functions $f_{q}(\mu), q=$ $1, \ldots, N$ and a constant $b_{0}>0$, such that the following statements are true when $\tau$ is large enough and

$$
|V| \exp \left(-b_{0} \tau \min \{L, t\}\right) \leqq 1
$$

(i) $f(\mu)=\min _{q} f_{q}(\mu)$.

(ii) $\left|\frac{d^{k}}{d \mu^{k}}\left(f_{q}(\mu)-e_{q}(\mu)\right)\right| \leqq O\left(e^{-\tau b_{0}}\right)$ if $|k| \leqq 4$

(iii) $\left|Z_{\mathrm{per}}(V, \mu)-\sum_{q} e^{-f_{q}(\mu)|V|}\right| \leqq e^{-f(\mu)|V|} e^{-b_{0} \tau \min \{L, t\}}$.

(iv) $\left|\frac{d^{k}}{d \mu^{k}}\left(M_{\mathrm{per}}(V, \mu)-\sum_{q} P_{q}(\mu) M_{q}(\mu)\right)\right| \leqq e^{-b_{0} \tau \min \{L, t\}}$,

where $|k| \leqq 3, P_{q}(\mu)=e^{-f_{q}(\mu)|V|}\left(\sum_{m} e^{-f_{m}(\mu)|V|}\right)^{-1}$, and $M_{q}(\mu)=-d f_{q}(\mu) / d \mu$.

Remarks. 1. Due to Theorem 2.1 (ii), the matrix

$$
F=\left(\frac{d}{d \mu_{i}}\left(f_{q}-f_{N}\right)\right)_{q, i=1, \ldots N-1}
$$

obeys a degeneracy removing condition of the form (2.7) as well. As a consequence, there is exactly one point $\mu^{*}$ such that $f_{q}(\mu)=f(\mu)$ for $\mu=\mu^{*}$. Using Theorem 2.1 (iv), we conclude that the infinite volume order parameter $M_{\text {per }}(\mu)=$ $\lim _{V \rightarrow \mathbf{Z}^{v+1}} M_{\text {per }}(V, \mu)$ is a convex combination of the order parameters $M_{q}$ with equal weight for all of them if $\mu=\mu^{*}$.

2. The explicit form of the finite-size scaling for the magnetization and other physically interesting quantities is obtained from Theorem 2.1 by expanding $f_{q}(\mu)$ around the coexistence point $\mu^{*}$,

$$
f_{q}(\mu)=f_{q}\left(\mu^{*}\right)+M_{q}^{i}\left(\mu^{*}\right)\left(\mu_{i}-\mu_{i}^{*}\right)+O\left(\left|\mu-\mu^{*}\right|^{2}\right) .
$$


3. In many models, the activities $\rho(Y)$ and the ground state energies $e_{m}$ are in fact analytic functions of $\mu$. While the "truncated free energies" $f_{q}(\mu)$ can probably be chosen $C^{\infty}$ in this case, we do not expect that they can be made analytic, since these would be analytic continuations of $f(\mu)$ across the point $\mu^{*}$, which are expected (and sometimes known, see [I]) not to exist.

We now proceed to the main focus of this paper, namely long volumes where (2.11) may not hold. In order to state the main results and to explain some of the ideas behind the proofs, we need the notion of a kink. We define: a contour $Y \subset V=A \times T$ is called short, if there is an interval $I \subset T$ of length $|I|<t=|T|$, such that $Y \subset A \times I$ (we use $I(Y)$ to denote the shortest interval $I$ for which this is true). Considering a short contour $Y$ as a subset of $V_{\infty}=A \times \mathbb{R}$, its complement has either one component or two infinite components. If it has two infinite components, it describes a transition from a state $m$ below $Y$ and a state $q$ above $Y$ (we sometimes emphasize this fact by a superscript $m q$ on $Y$ ) and is called a kink. Given a kink $Y$, we denote by $V(Y)$ the union of $Y$ with all finite components of $V_{\infty} \backslash Y$ and by $V_{+}\left(V_{-}\right)$the part of $(A \times I(Y)) \backslash V(Y)$ which lies above (below) $Y$.

The main ideas used in this paper can now be summarized as follows: in a first step, we rewrite the periodic partition function as a sum over kinks, resumming all other contours on the right-hand side of (2.3). This resummation has three effects: a "renormalization" of the ground state energy $e_{m}$ for the regions between kinks (we denote the new, effective ground state energy by $f_{m}(L)$ ), a "renormalization" of the kink activity $\rho(Y)$, and an effective interaction between two subsequent kinks. Neglecting this interaction, $Z_{\text {per }}(V)$ becomes

$$
Z_{\mathrm{per}}(Y) \approx \sum_{Y_{1}, \ldots, Y_{n}} \prod_{i} \kappa\left(Y_{i}\right) \prod_{i} e^{-f_{m_{1}}(L)\left|V_{i}\right|}
$$

where the sum goes over ordered sequences of kinks $Y_{i}=Y_{i}^{m_{i-1} m_{i}}, V_{i}$ is the region between $Y_{i}$ and $T_{i+1}$, and $\kappa\left(Y_{i}\right)$ is a modified kink activity (see Sect. 3 for the precise definitions of $f_{m}(L)$ and $\left.\kappa\right)$. In order to reduce the right-hand side of (2.14) to the partition function of a one-dimensional gas of kinks, we then associate an open interval $C(Y)$ of length one to each kink $Y$ (essentially, $C(Y)$ is the interval containing the middle point of $I(Y)$ ). Approximating the nonoverlap conditions on $Y_{1}, \ldots, Y_{n}$ by the condition that $C\left(Y_{i}\right)$ and $C\left(Y_{i+1}\right)$ do not overlap we obtain

$$
Z_{\text {per }}(V) \approx \sum_{C_{1}, \ldots, C_{n}} \prod_{i} r\left(C_{i}\right) e^{-f_{m_{1}}(L) L^{v} \Delta t_{i}}
$$

where $\Delta t_{i}$ is the distance between the upper end of $C_{i}$ and the lower end of $C_{i+1}$, and

$$
r\left(C_{i}\right)=\sum_{Y} \kappa(Y) e^{-f_{m_{-1}-1}(L)\left(|V-|-L^{\nu}|I-|\right)} e^{-f_{m_{i}}(L)\left(|V+|-L^{\nu}|I+|\right)} .
$$

Here $I_{ \pm}$is the upper (lower) part of $I(Y) \backslash C(Y), V_{ \pm}$are the volumes defined above, and the sum goes over kinks $Y=Y^{m_{i-1} m_{i}}$ such that $C(Y)=C_{i}$.

Due to the assumed translation invariance of our model, $r\left(C_{i}\right)$ does not in fact depend on the location of $C_{i}$ in $T$, but it does depend on the superscripts $m_{i-1} m_{i}$ of $C_{i}$. Introducing the matrices

$$
\Gamma_{m q}^{(1)}=\lim _{V \rightarrow V_{\infty}=A \times \mathbb{R}} r\left(C^{m q}\right)
$$


and

$$
F=\operatorname{diag}\left(e^{-|A| f_{m}(L)}\right),
$$

the right-hand side of $(2.15)$ can be written as a trace

$$
Z_{\mathrm{per}}(V) \approx \operatorname{tr}\left(\Gamma^{(1)}+F\right)^{t}=\sum_{i=1}^{N} \lambda_{i}^{(1)}(L)^{t},
$$

where $\lambda_{1}^{(1)}(L) \geqq \lambda_{2}^{(1)}(L) \geqq \cdots \lambda_{N}^{(1)}(L)$ are the eigenvalues of $\Gamma^{(1)}+F\left(\Gamma^{(1)}\right.$ is symmetric due to the reflection invariance of the model). There are several corrections to the above picture. First, we have neglected contributions from contours $Y$ to $Z_{\text {per }}$ which are not short; together with several other approximations made in the actual proof they contribute an error term

$$
L^{v} e^{-(\tau-O(1)) t} e^{-f|V|}
$$

Second, we neglected the interaction between kinks $Y_{i}, Y_{i+1}$. After a sequence of expansions, we are able to recover the expression (2.18), up to errors decaying rapidly with $t$ as in (2.19). However, the matrix $\Gamma^{(1)}$ becomes $\Gamma^{(1)}+\Gamma^{(2)}$, where $\Gamma^{2}$ involves at least two kinks, and hence is a very small change of order $e^{-(2 \tau-O(1)) L^{v}}$. The eigenvalues $\lambda_{1}(L), \ldots, \lambda_{N}(L)$ of $\Gamma^{(1)}+\Gamma^{(2)}+F$ are therefore close to $\lambda_{1}^{(1)}(L), \ldots, \lambda_{N}^{(1)}(L)$. And finally, the representation (2.14) is only a valid approximation if all phases are of "low energy" in the sense that $L^{v} a_{q} \leqq 7 \tau / 8$, say, where $a_{q}=f_{q}-\min _{m} f_{m}$.

In order to take this last effect into account, we artificially divide the states between low and high energy at a point where there is a gap. Certainly there is some $a^{*}$ with $L^{v} a^{*} \in[3 \tau / 4,13 \tau / 16]$ and such that no states $q$ lie in the range $a_{q} L^{v} \in\left[a^{*} L^{v}, a^{*} L^{v}+\tau /(16 N)\right]$. If $m$ is the first state above the gap, then $\tau^{*}=\min \left\{\tau, L^{v} a_{m}\right\}$ defines a decay rate associated with the high energy states. We denote the states below the gap by $Q_{s}(L)$; for these $L^{v} a_{q} \leqq 7 \tau / 8$ and (2.14) is a good approximation. The high energy states are so heavily suppressed that two kinks bounding a region in one of these phases can be considered as bound into one new kink. Since $Q_{s}(L)$ depends on $\mu$ and we need to control $\mu$-derivatives, we should relax these definitions slightly to allow for overlapping neighborhoods in which $Q_{s}(L)$ is independent of $\mu$.

Repeating this process for all high energy volumes $V_{i}$, one obtains a representation of the form (2.14), where the high energy have disappeared, but some of the kinks $Y_{i}$ are actually molecules of several kinks. These terms are $O\left(e^{-(2 \tau-O(1)) L^{v}}\right)$ and so can be absorbed into $\Gamma^{(>1)}$. Thus $(2.18)$ is valid with $F^{(1)}+F$ replaced by the $\left|Q_{s}(L)\right| \times\left|Q_{s}(L)\right|$ matrix

$$
\left(\Gamma_{p q}^{(1)}+\Gamma_{p q}^{(2)}+F_{p q}\right)_{p, q \in Q_{s}(L)},
$$

and the approximation there means neglecting terms of order $L^{v} e^{-\left(\tau^{*}-O(1)\right) t} e^{-f|V|}$.

We summarize our results in the following theorem, leaving its proof to Sect. 3 (where we analyze the interactions between kinks) and Sect. 4 (where we reduce the partition function to an equivalent one-dimensional system and break it down into product form (2.18)).

Theorem 2.2. Let $\tau$ be sufficiently large. For each $L$, there exists $a \tau^{*} \in[3 \tau / 4, \tau)$ and corresponding low energy phases $Q_{s}(L)$. For $p, q \in Q_{s}(L)$, there are functions $\Gamma_{p q}(L)$ and 
$f_{q}(L)$ forming a symmetric matrix $\Gamma$ and a diagonal matrix $F=\operatorname{diag}\left\{\exp \left(-L^{v} f_{q}(L)\right)\right\}$. Letting $\lambda_{1}(L) \geqq \cdots \geqq \lambda_{N(L)}(L)$ denote the (possibly repeated) eigenvalues of $F+\Gamma$, the following statements hold provided $|k| \leqq 4$ and

$$
L^{v} e^{-t} \leqq 1 \text {. }
$$

(i) asymptotics in $t$ :

$$
\left|\frac{d^{k}}{d \mu^{k}}\left[Z_{\mathrm{per}}(V)-\sum_{\alpha=1}^{N(L)} \lambda_{\alpha}(L)^{t}\right]\right| \leqq e^{-f|V|} e^{-\left(\tau^{*}-O(1)\right) t},
$$

(ii) comparison with $L=\infty$ :

$$
\left|\frac{d^{k}}{d \mu^{k}}\left(f_{q}(L)-f_{q}\right)\right| \leqq e^{-(\tau-O(1)) L},
$$

(iii) one-kink estimates on $\Gamma$ :

$$
\left|\frac{d^{k}}{d \mu^{k}} \Gamma_{p q}(L)\right| \leqq e^{-(f+\tau-O(1)) L^{v}} .
$$

(iv) Let $\Gamma^{(1)}(L)$ be the one-kink matrix defined in $(2.17 a)$. Then for $p, q \in Q_{s}(L)$,

$$
\left|\frac{d^{k}}{d \mu^{k}}\left[\Gamma_{p q}(L)-\Gamma_{p q}^{(1)}(L)\right]\right| \leqq e^{-f L^{v}} e^{-(2 \tau-O(1))\left(L^{v}-1 / 2\right)} .
$$

(v) Let $\lambda_{1}^{(1)}(L) \geqq \cdots \geqq \lambda_{N(L)}^{(1)}(L)$ be the eigenvalues of $F+\Gamma^{(1)}$. Relabeling if necessary, suppose that $Q_{s}(L)=\{1, \ldots, N(L)\}$ and $f_{1}(L) \leqq \cdots \leqq f_{N(L)}(L)$. Then $\lambda_{\alpha}(L)$ satisfy

$$
\begin{aligned}
\left|\lambda_{\alpha}(L)-\lambda_{\alpha}^{(1)}(L)\right| & \leqq e^{-f L^{\nu}-(2 \tau-O(1))\left(L^{\nu}-1 / 2\right)}, \\
\left|\lambda_{\alpha}(L)-\exp \left(-f_{\alpha}(L) L^{\nu}\right)\right| & \leqq e^{-(f+\tau-O(1)) L^{\nu}} .
\end{aligned}
$$

Remarks. 1. Statements (v) follow from (iv) by perturbation arguments. Similar statements hold for $\mu$-derivatives (with possible relabelings) but will contain small denominators if there are near-degeneracies in $\left\{\lambda_{\alpha}^{(1)}(L)\right\}$ or $\left\{f_{\alpha}(L)\right\}$. These statements show that a one-kink calculation is accurate up to two-kink effects. This of interest when combined with our asymptotics for $\Gamma_{p q}^{(1)}(L)$ in terms of surface tensions (derived in Sect. 5).

2. Since $\lambda_{1}(L)$ is close to $e^{-f_{1}(L) L^{\nu}}$, and since $f_{1}(L)=f+e^{-(\tau-O(1)) L}$ we have that

$$
\left(\frac{e^{-f L^{\nu}}}{\lambda_{1}(L)}\right)^{ \pm 1} \leqq \exp \left(e^{-(\tau-O(1)) L}\right) \leqq O(1) .
$$

We conclude that the bounds of Theorem 2.2 remain valid if the factors $e^{-f|V|}$ or $e^{-f L^{v}}$ on the right-hand sides of (i) (iii), (iv) and (v) are replaced by $\lambda_{1}(L)^{t}$ or $\lambda_{1}(L)$, respectively. Another consequence of $(v)$ is the positivity of $\lambda_{\alpha}(L)$ for all $\alpha$.

3. Theorem A follows from Theorem 2.2 since $\left|\mu-\mu^{*}\right| L^{v} \leqq 1$ implies that $a_{q} L^{v} \leqq$ $O(1)$. Then by construction $Q_{s}(L)=\{1, \ldots, N\}$. Thus we may take $M=F+\Gamma$.

Theorem 2.2 establishes a foundation for an analysis of scaling behavior of quantities such as magnetization. The following theorem describes what we know about the periodic magnetization $M_{\text {per }}(V, \mu)$ defines in (2.10). 
Theorem 2.3. Under the same conditions as in Theorem 2.2, let

$$
M_{\alpha}^{i}(L, \mu)=L^{-v} \frac{d}{d \mu_{i}} \log \lambda_{\alpha}(L)
$$

and

$$
P_{\alpha}(V, \mu)=\lambda_{\alpha}^{t}(L)\left[\sum_{\beta} \lambda_{\beta}^{t}(L)\right]^{-1}
$$

Then for $0 \leqq k \leqq 3$,

$$
\left|\frac{d^{k}}{d \mu^{k}}\left[M_{\mathrm{per}}^{i}(V, \mu)-\sum_{\alpha} M_{\alpha}^{i}(L, \mu) P_{\alpha}(V, \mu)\right]\right| \leqq e^{-\left(\tau^{*}-O(1)\right) t} .
$$

This result is an immediate consequence of Theorem 2.2. The interpretation is that each eigenvalue contributes $M_{\alpha}^{i}$ to the magnetization, but only as part of a convex combination determined by the $P_{\alpha}$. The largest eigenvalues receive the greatest weight, particularly for large $t$. In the cylindrical scaling regime, $t \rightarrow \infty$, the $P_{\alpha}$ trivialize to 0 or 1 while the $M_{\alpha}^{i}$ scale. In the block regime $L \sim t \rightarrow \infty$, the $M_{\alpha}^{i}$ are constants while the $P_{\alpha}$ scale. In the crossover regime both quantities scale nontrivially.

The next set of results represent an attempt to relate the off-diagonal matrix elements $\Gamma_{p q}(L)$ to the surface tension $\sigma_{p q}$ between phases $p$ and $q$, and to exhibit the dependence on $L$. These results depend on some additional assumptions on the structure of kinks separating to phases. Essentially we need that the kink activities factorize into contributions from each local excitation of the surface separating the two phases (Assumption 5.1). Each such contribution should decay exponentially in the size of the excitation (Assumption 5.2) and should depend on $\mu$ in a reasonable manner (Assumption 5.3). Furthermore, in dimension $v+1=2$, we need positive activities of excitation activities (Assumption 5.5). All these assumptions are reasonable for finite-range models of statistical mechanics at low temperature. The remaining Assumption 5.4 is a strict form of a triangle inequality for the surface tensions. Needed only if $N>2$, it allows us to neglect two-kink effects in comparison to one-kink effects. Thus it guarantees that $\Gamma_{p q}(L) \approx \Gamma_{p q}^{(1)}(L)$ (Proposition 5.3).

Theorem 2.4. Let $\tau$ be sufficiently large and assume that Assumptions 5.1 through 5.5 are valid. Define $a(\mu)=\max \left\{a_{q}(\mu), a_{p}(\mu)\right\}$. Then the following statements are true for a suitable constant $b_{1}>0, p \neq q$ and $L>1$.

(i) If the phases corresponding to $p$ and $q$ are stable (i.e. $a(\mu)=0$ )

$$
\begin{array}{ll}
\Gamma_{p q}(L)=\left(1+O\left(e^{-b_{1} \tau L}\right)\right) e^{-\sigma_{p q} L^{v}} e^{-f L^{v}}, & v \geqq 2, \\
\Gamma_{p q}(L)=\left(1+O\left(L^{-1}\right)\right) C_{p q} L^{-1 / 2} e^{-\sigma_{p q} L^{v}} e^{-f L^{v}}, & v=1, \quad L \gg 1,
\end{array}
$$

where $C_{p q}>0$ is a constant.

(ii) For $1 \leqq k \leqq 4$ and $a(\mu) L^{v} \leqq O(1)$,

$$
\left|\frac{d^{k} \Gamma_{p q}}{d \mu^{k}}\right| \leqq e^{O(1) L} \Gamma_{p q}
$$


(iii) For $v \geqq 2,1 \leqq k \leqq 4$ and $a(\mu) L^{v} \leqq O(1)$ the bound (2.27) may be sharpened to

$$
\left|\frac{d^{k} \Gamma_{p q}}{d \mu^{k}}\right| \leqq O\left(L^{v k}\right) \Gamma_{p q}
$$

(iv) For $v=1, k=1$ and $a(\mu) L \leqq O(1)$ the bound (2.27) may be sharpened to

$$
\left|\frac{d \Gamma_{p q}}{d \mu}\right| \leqq O(L) \Gamma_{p q}\left\{1+\frac{\left|f_{p}-f_{q}\right|}{2} e^{L O\left(e^{\left.-b_{1^{\tau}}\right)}\right.}\right\} .
$$

This theorem is proven at the end of Sect. 5 .

Proof of Theorem B. The above bound (2.26) gives Theorem B for $\mu=\mu^{*}$. For $\left|\mu-\mu^{*}\right| L^{v} \leqq e^{-\tau L / 2}, a(\mu) L^{v} \leqq O(1)$ and we may use Theorem 2.4(ii). Integrating the bound (2.27) from $\mu^{*}$ to $\mu$, we obtain the bound

$$
\Gamma_{p q}(\mu)=\Gamma_{p q}\left(\mu^{*}\right)\left(1+e^{O(1))} O\left(\left|\mu-\mu^{*}\right|\right)\right)=\Gamma_{p q}\left(\mu^{*}\right)\left(1+O\left(e^{-(\tau / 2-O(1)) L}\right)\right) .
$$

Together with the bound (2.26) and the fact that $\left|f(\mu)-f\left(\mu^{*}\right)\right| \leqq O\left(e^{-\tau L / 2}\right)$ this implies Theorem B.

\section{Kinks and Contours}

The goal of this section is to rewrite the periodic partition function as a sum over "kinks" (describing transitions between regions dominated by different ground states) and to study the interaction between these kinks.

Before defining the notion of a kink, we divide the set of contours in $V=A \times T$ into long and short contours, where a short contour is a contour $Y$ for which one may find an interval $I \subset T$ of length $|I|<t$ such that $Y \subset A \times I$ (a short volume $W \subset V$ is defined in the same way). Obviously, each short contour $Y$ may be considered as a contour in the infinite cylinder $V_{\infty}=A \times \mathbb{R}$, and $V_{\infty} \backslash Y$ has either one or two infinite connected components. In the first case we call $Y$ an ordinary contour, and in the second case we call it a kink.

In order to continue we need some notation: Given a short contour $(Y, q(\cdot))$, we define its interior, Int $Y$, as the union of all finite components of $V_{\infty} \backslash Y$, introduce $V(Y)=Y \cup$ Int $Y$, and let $\operatorname{Int}_{m} Y$ denote the union of all components $C$ of Int $Y$ for which $q(\cdot)$ takes the value $m$ on $\partial C$. If $Y$ is an ordinary contour, $V_{\infty} \backslash Y$ has only one infinite component. We call its boundary the external boundary of $Y$ and call $Y$ a $q$-contour if $q(\cdot)=q$ on the external boundary of $Y$. We sometimes emphasize the fact $Y$ is a $q$-contour by a superscript $q$ on $Y$. If $Y$ is a kink, one of the infinite components of $V_{\infty} \backslash Y$ contains the set $A \times\left[t_{0}, \infty\right)$ for all large enough $t_{0} \geqq 0$, while the other one contains the set $A \times\left(-\infty,-t_{0}\right]$. We call the boundaries of these components the external boundaries of $Y$ and use $\partial_{+} Y$ to denote the boundary of the infinite component which contains $A \times\left[t_{0}, \infty\right)$ and $\partial_{-} Y$ for the boundary of the component which contains $A \times\left(-\infty, t_{0}\right]$. If $q(\cdot)$ is $q$ on $\partial_{-} Y$ and $m$ on $\partial_{+} Y$ we say that $Y$ describes a transition from $q$ to $m$ and sometimes emphasize this by putting a superscript $q m$ on $Y$.

Finally, given a set of nonoverlapping ordinary contours $\left\{Y_{\alpha}\right\}$ we say that $Y$ is an external contour of $\left\{Y_{\alpha}\right\}$ if it is not contained in Int $Y_{\alpha}$ for any $\alpha$. 
Given these definitions we now rewrite $\mathrm{Z}_{\text {per }}(V)$ as

$$
Z_{\text {per }}(V)=\sum_{\left\{Y_{\alpha}\right\}}^{\prime} \prod_{\alpha} \rho\left(Y_{a}\right) \prod_{m} Z_{m}\left(V_{m}\right)
$$

where the sum $\sum^{\prime}$ goes over sets $\left\{Y_{\alpha}\right\}$ containing long contours and kinks, but no ordinary contour. As before any component of $V \backslash U_{\alpha} Y_{\alpha}$ has constant boundary conditions. $V_{m}$ denotes the union of those components which have boundary condition $m$, and (for any $W \subset V), Z_{m}(W)$ is defined by restricting the sum in (2.3) to a sum over collections $\left\{Y_{\alpha}\right\}$ of ordinary contours $Y \subset W$ whose exterior contours are $q$-contours. If $W^{c}=V \backslash W$ is not connected, we do not allow contours whose interior intersects $W^{c}$.

Obviously, the interaction between different kinks (and between kinks and long contours) is given by $Z_{m}\left(V_{m}\right)$. We therefore have to study the behavior of $Z_{q}(W)$ for $W \subset V, q=1, \ldots, N$. Fixing, for a moment, the external contours in the representation (2.3) for $Z_{q}(W)$ and resumming all the others we obtain a factor $\prod_{m} Z_{m}\left(\operatorname{Int}_{m} Y^{q}\right)$ for each external contour. This yields the expression

$$
Z_{q}(W)=\sum_{\left\{Y_{\alpha}^{q_{3}}\right\}_{\mathrm{ext}}} e^{-e_{q}\left|W \backslash \cup_{\alpha} V\left(Y_{\alpha}\right)\right|} \prod_{\alpha}\left[\rho\left(Y_{\alpha}^{q}\right) \prod_{m} Z_{m}\left(\operatorname{Int}_{m} Y_{\alpha}^{q}\right)\right]
$$

where the sum goes over sets $\left\{\mathrm{Y}_{\alpha}^{q}\right\}_{\text {ext }}$ of mutually external $q$-contours (that is $V\left(Y_{\alpha}\right)$ and $V\left(Y_{a}\right)$ have no common cubes for $\alpha \neq a$ ). We divide each $Z_{m}$ by the corresponding $Z_{q}$ and multiply it back again. Iterating this procedure we get

$$
Z_{q}(W)=e^{-e_{q}|W|} \sum_{\left\{Y_{\alpha}^{q}\right\}} \prod_{\alpha} K\left(Y_{\alpha}^{q}\right)
$$

where the sum goes over sets $\left\{Y_{\alpha}^{q}\right\}$ of $q$-contours in $W$ with the only restriction that $Y_{\alpha}^{q}$ and $Y_{a}^{q}$ have no common cubes for $\alpha \neq a$; and $K\left(Y^{q}\right)$ is defined by

$$
K\left(Y^{q}\right)=\rho\left(Y^{q}\right)^{e_{q}\left|Y^{q}\right|} \prod_{m} \frac{Z_{m}\left(\operatorname{Int}_{m} Y^{q}\right)}{Z_{q}\left(\operatorname{Int}_{m} Y^{q}\right)} .
$$

Note that (3.3) is purely formal at this moment since $Z_{q}\left(\operatorname{Int}_{m} Y^{q}\right)$ might be zero for some contours $Y^{q}$. The main content of the next lemma is that this does not happen if

$$
|\pi(Y)| a_{q} \leqq \frac{7}{8} \tau
$$

Here $\pi$ denotes the orthogonal projection from $V=A \times T$ onto $A,|\pi(Y)|$ is the $v$-dimensional volume of $\pi(Y)$, and

$$
a_{q}=f_{q}-f
$$

where $f=f(\mu)$ is the free energy and $f_{q}=f_{q}(\mu)$ are the functions introduced in Theorem 2.1 (see also Appendix A). Since the proof of this lemma is essentially the same as that of Lemma A.2 proven in the appendix we omit it here.

Lemma 3.1. There is a constant $b_{0}>0$ such that the following statements are true provided $\tau$ is large enough: 
(i) For all $|k| \leqq 4$, all $q \in Q$ and all short volumes $W$

$$
\left|\frac{d^{k}}{d \mu^{k}} Z_{m}(W)\right| \leqq \text { const }|W|^{k} e^{-f|W|+|\partial W|} \exp \left\{|W| e^{-b_{0} \tau L}\right\} .
$$

(ii) If $Y^{q}$ fulfills the condition (3.5) and $|k| \leqq 4$,

$$
\left|\frac{d^{k}}{d \mu^{k}} K\left(Y^{q}\right)\right| \leqq e^{-\left(\tau-a_{q}\left|\pi\left(Y^{q}\right)\right|-O(1)\right)\left|Y^{q}\right|} .
$$

(iii) If $W \subset V$ is chosen in such a way that all q-contours in $W$ fulfill the condition (3.5), then $Z_{q}(\tilde{W}) \neq 0$ for all $\tilde{W} \subset W$ and

$$
Z_{q}(W)=e^{-e_{q}|W|} \sum_{\left\{Y_{z}^{q^{q}}\right\}} \prod_{\alpha} K\left(Y_{\alpha}^{q}\right) .
$$

Remark. The factor $\exp \left\{|W| e^{-b_{0} \tau L}\right\}$ in Lemma 3.1, which is not present in the corresponding lemma in the appendix comes from the topologically nontrivial contours which are not present for the volumes considered in the appendix. Note $|W| e^{-L b_{0} \tau}$ can be bounded by $|\partial W|$ if $W$ is a short volume with connected boundary (as it is always the case of $W=\operatorname{Int}_{m} Y^{q}$ for some ordinary contour $Y^{q}$ ), since $|W| \leqq|\partial W||\pi(W)| \leqq|\partial W| L^{\nu}$.

We are now able to analyze the interaction between kinks in (3.1). In a first step we separate $Z_{\text {per }}(V)$ as given by (3.1) into a sum containing no long contour, henceforth denoted $Z_{\text {res }}(V)$, and a sum containing at least one long contour, $Z_{\text {big }}(V)$

$$
Z_{\text {per }}(V)=Z_{\text {big }}(V)+Z_{\text {res }}(V) \text {. }
$$

In the next step we prove a lemma bounding $Z_{\text {big }}(V)$ :

\section{Lemma 3.2.}

$$
\left|\frac{d^{k}}{d \mu^{k}} Z_{\mathrm{big}}(V)\right| e^{f|V|} \leqq L^{v} e^{-(\tau-O(1)) t}
$$

provided $|k| \leqq 4$ and

$$
L^{v} e^{-(\tau-O(1)) t} \leqq 1
$$

Proof. We rewrite $Z_{\text {big }}$ in the form

$$
Z_{\mathrm{big}}(V)=\sum_{n=1}^{\infty} \sum_{m=0}^{\infty} \frac{1}{n !} \frac{1}{m !} \sum_{Y_{1}, \ldots, Y_{n+m}} \prod_{i} \rho\left(Y_{i}\right) \sum_{M} Z_{m}\left(V_{m}\right)
$$

where $Y_{1}, \ldots, Y_{n}$ are long contours and $Y_{n+1}, \ldots, Y_{m+n}$ are kinks. Inserting Lemma 3.1 (i) and using the fact that $\left|f-e_{0}\right| \leqq O(1)$ to bound

we obtain that

$$
\begin{aligned}
\prod_{i}\left|\rho\left(Y_{i}\right)\right| \prod_{m} e^{-f\left|V_{m}\right|+\left|\partial V_{m}\right|} & =e^{-f|V|} \prod_{i}\left|\rho\left(Y_{i}\right)\right| e^{f\left|Y_{i}\right|+\left|\partial Y_{i}\right|} \\
& \leqq e^{-f|V|} \prod_{i} e^{-(\tau-O(1))\left|Y_{i}\right|}
\end{aligned}
$$

$$
\left|Z_{\mathrm{big}}(V)\right| \leqq \text { const } e^{-f|V|} \exp \left\{t L^{v} e^{-L \tau b_{0}}\right\} \sum_{n \geqq 1} \sum_{m \geqq 0} \frac{1}{n !} \frac{1}{m !} \sum_{Y_{1}, \ldots, Y_{n+m}} \prod_{i} e^{-(\tau-O(1))\left|Y_{i}\right|}
$$


Releasing the compatibility constraints on the contour sum and bounding $L^{v} e^{-L \tau b_{0}}$ by one we obtain

$$
\begin{aligned}
\mid Z_{\text {big }}(V) & \leqq \text { const } e^{t-f|V|}\left\{\exp \left(\sum_{Y:|Y| \geqq t} e^{-(\tau-O(1))|Y|}\right)-1\right\} \exp \left(\sum_{Y:|Y| \geqq L^{\nu}} e^{-(\tau-O(1))|Y|}\right) \\
& \leqq \text { const } e^{-f|V|} e^{t}\left\{\exp \left(t L^{v} e^{-(\tau-O(1)) t}\right)-1\right\} \exp \left(t L^{v} e^{-(\tau-O(1)) L^{\nu}}\right),
\end{aligned}
$$

which may be bounded by $L^{v} e^{-f|v|} e^{-(\tau-o(1)) t}$ if the condition (3.8) is true. The bounds on derivatives are similar and left to the reader.

Next we consider $Z_{\text {res }}(V)$. Obviously, $Z_{\text {res }}(V)$ can be rewritten as

$$
Z_{\mathrm{res}}(V)=\sum_{n \geqq 0} \frac{1}{n_{Y_{1}}, \ldots, Y_{n}} \sum_{i=1}^{n} Z_{q\left(V_{i}\right)}\left(V_{i}\right) \prod_{i=1}^{n}\left[\rho\left(Y_{i}\right) \prod_{m} Z_{m}\left(\operatorname{Int}_{m} Y^{i}\right)\right],
$$

where the sum goes over ordered sequences of nonoverlapping kinks $Y_{1}, \ldots, Y_{n}$ such that for each $i \in\{1, \ldots, n\}, \partial_{+} Y_{i} \cup \partial_{-} Y_{i+1}$ is the boundary of a maximally connected component $V_{i}$ of $V \backslash \bigcup_{i}\left\{Y_{i} \cup\right.$ Int $\left.Y_{i}\right\}$ (we identify $Y_{n+1}$ with $Y_{1}$ and also require boundary condition compatibility on $\partial Y_{i}$, that is $q\left(V_{i}\right)=q$ and $q\left(V_{i-1}\right)=m$ if $\left.Y_{i}=Y_{i}^{m q}\right)$. The factor $1 / n$ compensates for the fact $Y_{1}, \ldots, Y_{n}$ and $Y_{i+1}, Y_{i+2}, \ldots$, $T_{i-1}, Y_{i}$ correspond to the same set of kinks in the original sum (3.1) and should be interpreted as 1 if $n=0$ (in which case there are actually $N$ terms $Z_{q}(V)$, $q=1, \ldots, N)$.

Leaving the discussion of the general case to the end of this section, we will for the moment assume that all states $q \in Q$ lie below the gap introduced in Sect. 2. As a consequence,

$$
a_{q} L^{v} \leqq \frac{7}{8} \tau
$$

for all $q \in Q,(3.5)$ is fulfilled for all ordinary contours in $V$, and $Z_{q}(W)$ can be analyzed by a convergent cluster expansion for all $q \in Q$, all $W \subset V$ and all $V=A \times T$, uniform in $t=|T|$. We conclude that the finite $L$ free energy

$$
f_{q}(L)=-\lim _{V \rightarrow V_{\propto}=A \times \mathbb{R}} \frac{1}{|V|} \log Z_{q}(V)
$$

exists and obeys a bound

$$
\left|f_{q}(L)-f_{q}\right| \leqq e^{-\left(\tau-a_{q} L^{v}-O(1)\right) L} .
$$

In addition, for each $V_{i}$ contributing to (3.9)

$$
\log Z_{q}\left(V_{i}\right)=-f_{q}(L)\left|V_{i}\right|+\sum_{X} k_{q}(X)
$$

where the sum goes over connected subsets $X \subset V_{\infty}$ intersecting $\partial V_{i}=\partial_{+} Y_{i} \cup \partial_{-} Y_{i+1}$ and

$$
\left|k_{q}(X)\right| \leqq e^{-\left(\tau-a_{q} L^{v}-O(1)\right)|X|} .
$$

Setting $g\left(\partial_{+} Y_{i}\right)$ and $g\left(\partial_{i} Y_{i-1}\right)$ to the sum of all terms associated to the lower and upper boundary of $V_{i}$, respectively, we may rewrite

$$
\log Z_{q}\left(V_{i}\right)=-f_{q}(L)\left|V_{i}\right|+g_{q}\left(\partial_{+} Y_{i}\right)+g_{q}\left(\partial_{-} Y_{i+1}\right)+g_{q}\left(Y_{i}, Y_{i+1}\right),
$$


where the interaction term $g_{q}\left(Y_{i}, Y_{i+1}\right)$ can be written as a sum over connected sets $X$ intersecting both $\partial_{-} Y_{i+1}$ and $\partial_{+} Y_{i}$. As usual

$$
\left|g_{q}\left(\partial_{ \pm} Y\right)\right| \leqq\left|\partial_{ \pm} Y\right| O\left(e^{-\left(\tau-a_{q} L^{\nu}\right)}\right)
$$

and

$$
\left|g_{q}\left(Y_{i}, Y_{i+1}\right)\right| \leqq \min \left\{\left|\partial_{+} Y_{i}\right|,\left|\partial_{-} Y_{i+1}\right|\right\} e^{-\left(\tau-a_{q} L^{v}-O(1)\right)\left(\operatorname{dist}\left(Y_{i}, Y_{i+1}\right)+1\right)}
$$

where we used (3.13) and the fact that the number of connected sets $X$ of size $|X|=s$ which intersect a given set $M$ can be bounded by $|M|$ const $^{s}$. Combining (3.9) and (3.14) we get

$$
Z_{\mathrm{res}}(V)=\sum_{n \geqq 0} \frac{1}{n_{Y_{1}}, \ldots, Y_{n}} \sum\left[\kappa\left(Y_{i}\right) e^{-f_{q_{i}}(L)\left|V_{i}\right|}\right] e^{g_{q_{i}}\left(Y_{i}, Y_{i+1}\right)},
$$

where we used the abbreviations

$$
\kappa\left(Y^{m q}\right)=\rho\left(Y^{m q}\right) e^{g_{m}\left(\partial-Y^{q m}\right)+g_{q}\left(\partial_{+} Y^{m q}\right)} \sum_{\tilde{m}} Z_{\tilde{m}}\left(\operatorname{Int}_{\tilde{m}} Y^{m q}\right)
$$

and $q_{i}=q\left(V_{i}\right)$.

Remarks. 1. The leading term in $n$ in (3.17) comes from the sum

$$
\sum_{q \in Q} Z_{q}(V)
$$

and should therefore be written as

$$
\sum_{q \in Q} \exp \left\{-f_{q}(L)|V|+O\left(|V| e^{-t\left(\tau-a_{q} L^{\nu}-O(1)\right)}\right)\right\} .
$$

Assuming that a condition of the form

$$
L^{v} e^{-(\tau / 8-O(1)) t} \leqq 1
$$

is true, $|V| e^{-t\left(\tau-a_{q} L^{v}-O(1)\right)} \leqq 1$ and the sum $\sum_{q} Z_{q}(V)$ differs from the corresponding term $\sum_{q} \exp \left\{-f_{q}(L)|V|\right\}$ by a number which may be bounded by

$$
\begin{aligned}
\sum_{q \in Q} e^{-f_{q}(L)|V|}\left|\exp \left\{O\left(|V| e^{-t\left(\tau-a_{q} L^{\nu}-O(1)\right)}\right)\right\}-1\right| & \leqq \sum_{q \in Q} e^{-f_{q}(L)|V|} O\left(|V| e^{-t\left(\tau-a_{q} L^{v}-O(1)\right)}\right) \\
& \leqq L^{v} e^{-t(\tau-O(1))} \sum_{q \in Q} e^{\left(f_{q}-f_{q}(L)\right)|V|} e^{-f|V|} \\
& \leqq L^{v} e^{-t(\tau-O(1))} e^{-f|V|}
\end{aligned}
$$

We have left out this correction in (3.17) and assume it has been absorbed into $Z_{\text {big }}(V)$.

2. By Lemma 3.1

$$
\begin{aligned}
\left|Z_{\tilde{m}}\left(\operatorname{Int}_{\tilde{m}} Y^{m q}\right)\right| & \leqq \exp \left(-f\left|\operatorname{Int}_{\tilde{m}} Y^{m q}\right|+O(1)\left|Y^{m q}\right|\right) \\
& \leqq \exp \left(-f(L)\left|\operatorname{Int}_{\tilde{m}} Y^{m q}\right|+O(1)\left|Y^{m q}\right|\right),
\end{aligned}
$$


where we used the bound (3.12) and the fact that $\left|\operatorname{Int}_{\tilde{m}} Y^{m q}\right| \leqq L^{v}\left|Y^{m q}\right|$ to replace $f$ by

$$
f(L)=\min _{q} f_{q}(L)
$$

in the last step. Combining the above bound with the a priori bound on $\rho$, the bound (3.15), and the fact that $\left|e_{0}-f(L)\right| O(1)$ one obtains that

$$
|\kappa(Y)| \leqq e^{-(\tau-O(1))|Y|} e^{-f(L)|V(Y)|}
$$

Generalizing this to derivatives we get

$$
\left|\frac{d^{k}}{d \mu^{k}} \kappa(Y)\right| \leqq e^{-(\tau-O(1))|Y|} e^{-f(L)|V(Y)|}
$$

for all multi-indicates $k$ of order $|k| \leqq 4$.

We finally consider the case where some of the sets $m \in Q$ lie above the gap. Since $Z_{m}\left(V_{i}\right)$ can in general not be analyzed by a convergent expansion for such an $m$, we leave it as it is and replace (3.17) by

$$
Z_{\mathrm{res}}(V)=\sum_{n \geqq 0} \frac{1}{n_{Y_{1}}, \ldots, Y_{n}} \prod_{i}\left[\kappa\left(Y_{i}\right) Z_{q_{i}}\left(V_{i}^{u}\right) e^{-f_{q_{i}}(L)\left|V_{q}^{s}\right|} \prod_{i} e^{g_{q_{i}}\left(Y_{i}, Y_{i+1}\right)}\right]
$$

where we used the superscript $u$ (=unstable) for the volumes $V_{i}$ corresponding to states $q$ which lie above the gap and $s\left(=\right.$ stable) for all others. That is $V_{i}^{u}=V_{i}$ and $V_{i}^{s}=\varnothing$ if $q\left(V_{i}\right)$ is above the gap while $V_{i}^{u}=\varnothing$ and $V_{i}^{s}=V_{i}$ if $q\left(V_{i}\right)$ lies below the gap. Obviously, we also have to change the definition of $\kappa$ and the interaction term $g(\cdot, \cdot)$ by defining

$$
g_{m}(\cdot)=g_{m}(\cdot, \cdot)=0
$$

if $m$ lies above the gap.

In order to state the next lemma, we introduce, for $W \subset V$, the number $t(W)$ as the size of the maximal subset $S \subset T$ such that $A \times S \subset W$.

Lemma 3.3. Assume that

$$
L^{v} e^{-t} \leqq 1
$$

and that $|k| \leqq 4$. Then

$$
\left|\frac{d^{k}}{d \mu^{k}} Z_{m}(W)\right| \leqq|W|^{|k|} e^{-f|W|+O(1)|\partial W|} e^{-t(W)\left(\min \left\{\tau, a_{m} L^{v}\right\}-O(1)\right)}
$$

Proof. The proof of Lemma 3.3 is essentially identical to the proof of Lemma 5.4 in [BI1]. We define an ordinary $m$-contour to be small if $a_{m}|\pi(Y)| \leqq 7 \tau / 8$ and use the relation (3.2) to rewrite $Z_{m}(W)$ in the following way. Write a set $\left\{\bar{Y}_{\alpha}^{m}\right\}$ of external $m$-contours in $W$ as $\left\{X_{\alpha}^{m}\right\} \cup\left\{Z_{\alpha}^{n}\right\}$, where $\left\{Z_{\alpha}^{m}\right\}$ denote the small contours in $\left\{Y_{\alpha}^{m}\right\}$ and $\left\{X_{\alpha}^{m}\right\}$ the large contours in $\left\{Y_{\alpha}^{m}\right\}$. Note that for fixed $X_{\alpha}^{q}$ 's, the sum over $\left\{Z_{\alpha}^{q}\right\}$ goes over all sets of mutually external small contours in Ext $=W \backslash \bigcup_{\alpha} V\left(X_{\alpha}\right)$. Thus, resumming the small contours and using (3.2) a second time,

$$
Z_{m}(W)=\sum_{\left\{X_{\alpha}^{m}\right\}_{\text {ext }}} Z_{m}^{\text {small }}(\mathrm{Ext}) \prod_{\alpha}\left[\rho\left(X_{\alpha}^{m}\right) \prod_{q} Z_{q}\left(\mathrm{Int}_{q} X_{\alpha}^{m}\right)\right]
$$


where the sum goes over sets of mutually external large contours in $W$ and $Z_{M}^{\text {small }}\left(\right.$ Ext) is obtained from $Z_{m}^{\text {small }}($ Ext) by dropping all large external $m$-contours.

By Lemma 3.1(ii), $\log Z_{m}^{\text {small }}($ Ext) can be analyzed by a convergent cluster expansion. Comparing this expression to the expansion of $f_{m} \mid$ Ext $\mid$ one finds that

$$
\left|f_{m}\right| \operatorname{Ext}\left|+\log Z_{m}^{\text {small }}(\operatorname{Ext})\right| \leqq|\partial \operatorname{Ext}| O\left(e^{-\tau b_{0}}\right)+|\operatorname{Ext}| e^{-\tau b_{0} \min \{L, t\}}+|\operatorname{Ext}| e^{-\tau^{2} b_{0} / a_{m}},
$$

where the first item is the usual boundary term, the second comes from the topologically nontrivial excitations in Ext and the third comes from the fact that the large contours which are truncated in $Z_{m}^{\text {small }}$ still contribute to $f_{m}$ (the factor $\tau^{2} / a_{m}$ arises because each large contour has a size $|Y| \geqq O\left(\tau / a_{m}\right)$, and contours are suppressed like $\exp (-\tau O(|Y|))$.) Using the fact that

$$
|\partial \operatorname{ext}|=|\partial W|+\sum_{\alpha}\left|\partial X_{\alpha}^{m}\right| \leqq|\partial W|+2 d \sum_{\alpha}\left|X_{\alpha}^{m}\right|,
$$

we now bound

$$
\begin{aligned}
\left|Z_{m}^{\text {small }}(\mathrm{Ext}) e^{f|\mathrm{Ext}|}\right| \leqq & \exp \left\{|\partial W| O\left(e^{-\tau b_{0}}\right)+\sum_{\alpha} 2 d\left|X_{\alpha}^{m}\right|+|W| e^{-\tau b_{0} \min \{L, t\}}\right\} \\
& \cdot \exp \left\{-\left(a_{m}-e^{-\tau^{2} b_{0} / a_{m}}\right)|\mathrm{Ext}|\right\} \\
\leqq & \exp \left\{|\partial W| O\left(e^{-\tau b_{0}}\right)+\sum_{\alpha} 2 d\left|X_{\alpha}^{m}\right|+|W| e^{-\tau b_{0} \min \{L, t\}}\right\} \\
& \cdot \exp \left\{-\left(a_{m}\left(1-\frac{b_{0}}{\tau^{2}}\right)\right)|\mathrm{Ext}|\right\} .
\end{aligned}
$$

Inserting this bound, the bound (i) of Lemma 3.1, and the bound

$$
\left|\rho\left(X_{\alpha}\right)\right| \leqq e^{-\left(\tau+e_{0}\right)\left|X_{q}\right|} \leqq e^{-\left(\tau+f-O\left(e^{-b_{0} \tau}\right)\right)\left|X_{\alpha}\right|}
$$

into (3.22) we obtain

$$
\begin{aligned}
\left|Z_{m}(W)\right| e^{f|W|} \leqq & \exp \left\{|\partial W| O\left(e^{-\tau b_{0}}\right)+|W| e^{\tau b_{0} \min \{L, t\}}\right\} \\
& \cdot \sum_{\left\{X_{\alpha}^{m}\right\}_{\text {ext }}} e^{-a_{m}\left(1-b_{0} \tau^{-2}\right)|E x t|} \prod_{\alpha} e^{-(\tau-O(1))\left|X_{\alpha}^{m}\right|} .
\end{aligned}
$$

Extracting a factor

$$
\max _{\left\{X_{\alpha}^{m}\right\}_{\text {ext }}} e^{-a_{m}\left(1-O\left(\tau^{-1}\right)\right)|\operatorname{Ext}|} \prod_{\alpha} e^{-(\tau-O(1))\left|X_{\alpha}^{m}\right|}
$$

and bounding the remaining sum as in the proof of Theorem 3.1 (ii) in [BI1] we get

$$
\begin{aligned}
\left|Z_{m}(W)\right| e^{f|W|} & \leqq \exp \left\{|\partial W|+|W| e^{\min \{L, t\}}\right\} \max _{\left\{X_{\alpha}^{m}\right\}} e^{-a_{m}\left(1-O\left(\tau^{-1}\right)\right)|\operatorname{Ext}|} \prod_{\alpha} e^{-(\tau-O(1))\left|X_{\alpha}^{m}\right|} \\
& \leqq \exp \{|\partial W| O(1)+t(W) O(1)\} \max _{\left\{X_{\alpha}^{m}\right\}} e^{-a_{m}\left(1-O\left(\tau^{-1}\right)\right)|\operatorname{Ext}|} \prod_{\alpha} e^{-(\tau-O(1))\left|X_{\alpha}^{m}\right|},
\end{aligned}
$$

where we used the assumption $\left(3.8^{\prime \prime}\right)$ and the fact that $|W| \leqq(|\partial W|+t(W)) L^{v}$ in the last step. Now, each time slice in $A \times S$ either contributes a smaller factor

$$
e^{-a_{m}\left(1-O\left(\tau^{-1}\right)\right) L^{v}} \leqq e^{O(1)-\min \left\{\tau, \alpha_{m} L^{v}\right\}}
$$

to $e^{-a_{m}\left(1-O\left(\tau^{-1}\right)\right)|E x t|}$ or contains at least one cube in $\bigcup_{\alpha} X_{\alpha}^{m}$ and therefore contributes 
a small factor $\leqq e^{-(\tau-O(1))}$ to the product $\prod_{\alpha} e^{-(\tau-O(1))\left|X_{\alpha}\right|}$. As a consequence,

$$
e^{-a_{m}\left(1-O\left(\tau^{-1}\right)\right)|\mathrm{Ext}|} \prod_{\alpha} e^{-(\tau-O(1))\left|X_{\alpha}^{m}\right|} \leqq e^{\left(O(1)-\min \left\{\tau, a_{m} L^{\eta}\right\}\right)|S|}=e^{\left(O(1)-\min \left\{\tau, a_{m} L^{\eta}\right) t(W)\right.} .
$$

This completes the proof of (3.21) for $|k|=0$. The proof for the derivatives is similar and is left to the reader.

Remarks. 1. As before, the leading term in $n$ in $\left(3.17^{\prime}\right)$ comes from the sum

$$
\sum_{q \in Q} Z_{q}(V)
$$

but $Z_{q}(V) \approx e^{-f_{q}(L)|V|}$ is only true if $q$ lies below the gap. But for $q$ above the gap,

$$
Z_{q}(V) \leqq e^{-t\left(\tau^{*}-O(1)\right)} e^{-f|V|}
$$

due to Lemma 3.3 (recall that $\tau^{*}=\underset{q \notin Q_{s}(L)}{\min } \min \left\{\tau, a_{q} L v\right\}$ ). We have left out this term in $\left(3.17^{\prime}\right)$ and assume it has been absorbed into $Z_{\text {big }}(V)$.

2. The free energies $f_{q}(L)$ may be defined even if $a_{q} L^{v}>7 \tau / 8$. To this end one introduces truncated activities $K^{\prime}\left(Y^{q}\right)$ as in Appendix A, see Eq. (A.8), defines

$$
Z_{q}^{\prime}(V)=e^{-e_{q}|V|} \prod_{\left\{Y_{\alpha}^{q}\right\}} \prod_{\alpha} K^{\prime}\left(Y_{\alpha}^{q}\right)
$$

and takes the limit

$$
f_{q}(L)=-\lim _{V \rightarrow V_{\infty}} \frac{1}{|V|} \log Z_{q}^{\prime}(V) .
$$

The resulting $f_{q}(L)$ is a $C^{4}$-continuation of the $f_{q}(L)$ defined in (3.11) and

$$
\left|\frac{d^{k}}{d \mu^{k}}\left(f_{q}(L)-e_{q}\right)\right| \leqq O\left(e^{-(\tau / 8-O(1)) L}\right) .
$$

The logarithm of $Z_{q}^{\prime}\left(V_{i}\right)$ can be analyzed by a convergent expansion for all $\mu$ :

$$
\log Z_{q}^{\prime}\left(V_{i}\right)=-f_{q}(L)\left|V_{i}\right|+\sum_{X} k_{q}^{\prime}(X)
$$

(cf. Eq. (3.13a)) with

$$
\left|\frac{d^{k}}{d \mu^{k}} k_{q}^{\prime}(X)\right| \leqq e^{-(\tau / 8-O(1))|X|}
$$

Finally,

$$
k_{q}^{\prime}(X)=k_{q}(X) \quad \text { if } \quad a_{q} L^{v} \leqq \frac{7}{8} \tau
$$

\section{A One-Dimensional System of Rods}

In this section we reduce the kink partition function (3.17) to an equivalent one-dimensional system of hard-core rods, and then obtain the product form after a sequence of expansions. 
In a preliminary step we make the definition of the low energy states $Q_{s}(L)$ a little bit more precise. Given $L$ and $\tilde{\mu} \in R^{N-1}$, we chose $a^{*} \in L^{-v}[3 \tau / 4,13 \tau / 16]$ in such a way that no states $q$ lie in the range $a_{q}(\tilde{\mu}) L^{v} \in\left[a^{*} L^{v}, a^{*} L^{v}+\tau /(16 N)\right]$. We denote the states below the gap by $Q_{s}(L)=Q_{s}(L, \tilde{\mu})$ and define

$$
\tau^{*}(L, \mu)=\min \left\{\tau, \min _{m \notin Q_{s}(L, \tilde{\mu})} L^{v} a_{m}(L, \mu)\right\},
$$

where

$$
\begin{aligned}
a_{m}(L, \mu) & \equiv f_{m}(L, \mu)-\min _{q} f_{q}(L, \mu) \\
& =a_{m}(\mu)+O\left(e^{-b_{1} \tau L}\right)=a_{m}(\tilde{\mu})+O\left(L^{-v}\right)
\end{aligned}
$$

if $|\mu-\tilde{\mu}| L^{v}<1$. Throughout this section, $\tilde{\mu}$ and $Q_{s}(L)=Q_{s}(L, \tilde{\mu})$ are fixed, $|\mu-\tilde{\mu}| L^{v}<1$ and $\tau^{*}$ and $a_{m}$ denote $\tau^{*}(L, \mu)$ and $a_{m}(L, \mu)$, respectively. Note that

$$
\tau^{*} \geqq\left(\frac{3}{4}+\frac{1}{16 N}\right) \tau-O(1) \geqq \frac{3 \tau}{4}
$$

due to the above bound on $a_{m}(L, \mu)-a_{m}(\tilde{\mu})$.

After these preliminaries we expand

$$
e^{g_{q}\left(Y_{i}, Y_{i+1}\right)}=1+\tilde{g}_{q}\left(Y_{i}, Y_{i+1}\right)
$$

where by (3.16),

$$
\left|\tilde{g}_{q}\left(Y_{i}, Y_{i+1}\right)\right| \leqq e^{\left|Y_{i}\right|} e^{-\left(\tau-a_{q} L^{v}-O(1)\right)\left(\operatorname{dist}\left(Y_{i}, Y_{i}+1\right)+1\right)} .
$$

Each $\tilde{g}$ term is considered to connect $Y_{i}$ with $Y_{i+1}$. Also, $Y_{i}$ and $Y_{i+1}$ are considered connected if the lowest point of $Y_{i+1}$ is below the highest point of $Y_{i}$. Finally, $Y_{i}$ and $Y_{i+1}$ are considered connected if $V_{i}^{u} \neq \varnothing$. To any connected string $Y_{i}, \ldots, Y_{i+l}$ are associate an interval $I \subset T$ which extends from the lowest point of $Y_{i}$ to the highest point of $Y_{i+l}$. Thus, $I$ is a union of the unit intervals of $T$. We form the following activity:

$$
\begin{aligned}
r_{1}(I)= & \sum_{l \geqq 0} \sum_{Y_{0}, \ldots, Y_{l}} \prod_{j=0}^{l-1}\left[\kappa\left(Y_{j}\right) Z_{q_{j}}\left(V_{j}^{u}\right) e^{-f_{q_{j}}(L)\left|V_{j}^{s}\right|}\left\{\begin{array}{c}
\tilde{g}_{q_{j}}\left(Y_{j}, Y_{j+1}\right) \\
\text { or } \\
1+\tilde{g}_{q_{j}}\left(Y_{j}, Y_{j+1}\right)
\end{array}\right\}\right] \\
& \cdot \kappa\left(Y_{l}\right) e^{-f_{q_{-}}(L)|V-|-f_{q_{+}}(L) \mid V+1} .
\end{aligned}
$$

Here the sum is over compatible sequences of kinks, $Y_{0}, \ldots, Y_{l}$, whose spanning interval is $I$. The phase at the bottom of $Y_{0}$ is $q_{-}$, the one at the top of $Y_{l}$ is $q_{+}$. The regions $V_{-}\left(V_{+}\right)$are the portions of $A \times I$ below $Y_{0}$ (above $\left.Y_{l}\right)$. The term $\tilde{g}$ is selected if it is needed to connect $Y_{j}$ and $Y_{j+1}$, otherwise $1+\tilde{g}$ is selected.

These new kink activities allow us to write

$$
\begin{aligned}
Z_{\text {res }}(V) & =Z_{\text {res }, 1}(V)+Z_{\text {big, } 1}(V), \\
Z_{\text {res }, 1}(V) & =\sum_{n \geqq 0} \frac{1}{n_{I_{1}}, \ldots, I_{n}} \sum_{i=1}^{n}\left[r_{1}\left(I_{i}\right) e^{-f_{q_{i}}(L) L^{v}\left|J_{i}\right|}\right],
\end{aligned}
$$

where the second sum in (4.3) goes over open intervals $I_{1}, \ldots, I_{n}$ such that $I_{i}$ and 
$I_{j}$ do not overlap for $i \neq j$ and $Z_{\mathrm{big}, 1}(V)$ is the sum of terms where the interval formed by the construction above is all of $T$. Each interval in (4.3) is really a triple $\left(I, q_{+}, q_{-}\right)$, where $q_{+}\left(q_{-}\right)$is an assignment of a label in $Q$ to the upper (lower) endpoint of $I$. The interval $J_{i}$ is the one between $I_{i}$ and $I_{i+1}$; both endpoints of $J_{i}$ have the same label $q_{i}$. The length of $J$ is denoted $|J|$. Note that $q_{ \pm}(I)$ no longer take values corresponding to the high energy phases.

We give bounds on $r(I)$ by writing

$$
r_{1}(I)=r_{1}^{(1)}(I)+r_{1}^{(2)}(I)
$$

where $r_{1}^{(1)}$ is the one-kink term $(l=0$ in $(4.2))$ and $r_{1}^{(2)}$ is the rest. First, using the bound (3.20) on $\kappa$, we obtain

$$
\left|r_{1}^{(1)}(I)\right| \leqq e^{-(\tau-O(1))(|I|-1)} e^{-(\tau-O(1)) L^{v}} e^{-f(L) L^{\nu}|I|} .
$$

Here we use the fact that the minimum size of a kink is $L^{v}$. When we apply $\mu$-derivatives to $f_{q_{ \pm}}(L)$, we bring down factors $\left|V_{ \pm}\right| \leqq|I| L^{\nu}$, which can be absorbed into the constants above. Hence the same estimate holds for $d^{k} r^{(1)} / d \mu^{k}$. Better estimates should hold for $r_{1}^{(2)}$ since there are at least two kinks. But we have to bound

$$
Z_{q}\left(V^{u}\right) \leqq e^{-f\left|V^{u}\right|+O(1)\left|\partial V^{u}\right|} e^{-\left(\tau^{*}-O(1)\right) t\left(V^{u}\right)},
$$

which is Lemma 3.3. (Recall that $\tau^{*}=\min \left\{\tau, L^{v} a_{m}\right\}$, where $a_{m}$ is the lowest value amongst the high energy states.) We estimate $\tilde{g}_{q}$ using (4.1), only we settle for a decay $\exp \left[-\left(\tau-a_{q} L^{v}-O(1)\right) t\left(V_{i}^{s}\right)\right]$. Then each factor $\exp \left(-\tau+a_{q} L^{v}\right)$ from this bound combines with $\exp \left(-f_{q} L^{v}\right)$ from $V_{i}^{s}$ is to produce an overall $\exp \left[\left(-\tau-f(L) L^{v}\right) t\left(V_{i}^{s}\right)\right]$. Differences $\left|f_{q}-f_{q}(L)\right| L^{v}|I|$ are $O(1)|I|$ and are harmless. The parts of $I$ not covered by this argument get their decay from $\kappa$, as in the one-kink estimate (4.4). This includes volumes where 1 is selected in place of $\tilde{g}$. Summing over $Y_{0}, \ldots, Y_{l}$ yields only another harmless $\exp (O(1)|I|)$ factor. Summarizing, we obtain

$$
\left|r_{1}^{(2)}(I)\right| \leqq e^{-\left(\tau^{*}-O(1)\right)(|I|-2)} e^{-2(\tau-O(1)) L^{v}} e^{-f(L) L^{\nu}|I|} .
$$

Derivatives of $r_{1}^{(2)}$ again produce volume factors from differentiating $Z_{q}\left(V^{u}\right)$ or $f_{q}(L)$, but as in the one-kink estimate they can be absorbed into the constants in (4.6).

In a similar fashion we bound

$$
\left|Z_{\text {big }, 1}(V)\right| \leqq e^{-\left(\tau^{*}-O(1)\right) t} e^{-(\tau-O(1)) L^{v}} e^{-f|V|} .
$$

Since $Z_{\mathrm{big}}, Z_{\mathrm{big}, 1}$ decay so rapidly in $t$, we may focus attention on $Z_{\mathrm{res}, 1}$ which contains the main asymptotics.

The only obstruction to expressing $Z_{\mathrm{res}, 1}$ in product form is the presence of rods with $|I|>1$. In order to effectively shrink intervals to unit length, we make use of shrunken intervals $C$. We say $C$ is a core of $I$ if $C$ is a unit interval closest to the center of $I$ (two choices if $|I|$ is even; all intervals are built of unit intervals with integer centers). Our rod activities will be functions of a pair $(C, I)$ such that $C$ is a core of $I$. Define

$$
r_{1}(C, I)=\frac{2}{3+(-1)^{|I|}} r_{1}(I) \exp \left(f_{q_{+}}(L) L^{v}\left|I^{+}\right|+f_{q_{-}}(L) L^{\nu}\left|I^{-1}\right| .\right.
$$


Here $I^{+}$is the part of $I$ above $C$ and $I^{-}$is the part below $C$. The factor $\frac{2}{3+(-1)^{|I|}}$ is inserted, since if $|I|$ is even, two $C$ 's are possible. This activity obeys the following estimate:

$$
\left|r_{1}(C, I)\right| \leqq e^{-\left(\tau^{*}-a_{q_{+}} L^{v}-O(1)\right) \mid I^{+}}\left|e^{-\left(\tau^{*}-a_{q_{-}} L^{v}-O(1)\right) \mid I^{+}}\right| e^{-(\tau-O(1)) L^{v}} e^{-f(L) L^{v}}
$$

Here we take a bound covering both cases (4.4), (4.6). Multiplying the energy densities $f_{q_{ \pm}}(L)$ cancels most of the decay but due to the gap between the low and high energy phases, a decay $\Delta \tau$ in $|I|$ remains. Thus we take

$$
\Delta=\left(\tau^{*}-\max _{q \in Q_{s}(L)} a_{q}(L, \mu) L^{v}\right) \tau^{-1} \geqq \frac{1}{16 N} \rightarrow O(1 / \tau) .
$$

Now we sum over the collections of pairs $(C, I)$ in $Z_{\text {res, } 1}$ :

$$
Z_{\mathrm{res}, 1}(V)=\sum_{n \geqq 0} \frac{1}{n_{\left(C_{1}, I_{1}\right)}, \ldots,\left(C_{n}, I_{n}\right)} \prod_{i=1}^{n}\left[r_{1}\left(C_{i}, I_{i}\right) e^{-f_{q_{i}}(L) L^{\nu}\left|K_{i}\right|}\right] .
$$

Here $K_{i}$ is the interval between $C_{i}$ and $C_{i+1}$.

The next step is to expand part of the hard-core interactions between intervals, reducing each interval $I_{i}$ to its core $C_{i}$. To this end we extend the sum over $\left(C_{1}, I_{1}\right), \ldots,\left(C_{n}, I_{n}\right)$ to include overlapping intervals. We even permit $I_{i}$ to wrap around $T$; in this case the activity is obtained from the $t \rightarrow \infty$ limit, with $I_{i}$ any interval of $\mathbb{R}$. However, we maintain the following conditions:

(i) $C_{i} \cap C_{j}=\varnothing$ (as open intervals)

(ii) $C_{1}, \ldots, C_{n}$ are arranged cyclically around $T$ in order

(iii) $q_{+}\left(C_{i}\right)=q_{-}\left(C_{i+1}\right)$.

In the third condition, the phase $q_{ \pm}\left(C_{i}\right)$ are inherited from $I_{i}$. In order to recover $Z_{\text {res, } 1}(V)$, we insert a factor $\prod_{i=1}^{n} U\left(I_{i}^{+}, I_{i+1}^{-}\right)$, where

$$
U\left(I_{i}^{+}, I_{i+1}^{-}\right)= \begin{cases}0 & \text { if }\left(I_{i}^{+} \cup C_{i}\right) \cap\left(I_{i+1}^{-} \cup C_{i}\right) \neq \varnothing . \\ 1 & \text { otherwise }\end{cases}
$$

It is only necessary to enforce nonoverlapping conditions between neighboring intervals; the other conditions follow.

We now expand $U \equiv 1+A$ to obtain

$$
\prod_{i=1}^{n} U\left(I_{i}^{+}, I_{i+1}^{-}\right)=\sum \prod A
$$

For each $A\left(I_{i}^{+}, I_{i+1}^{-}\right)$present, we draw the open interval $I_{i}^{+} \cup I_{i+1}^{-}$. We also draw the open intervals $C_{i}$. The union of all lines drawn breaks into connected components, and these components form a new system of rods.

The activity $r_{2}(J)$ associated with one of these components is

$$
r_{2}(J)=\sum_{n \geqq 1} \sum_{\left(C_{1}, I_{1}\right), \ldots,\left(C_{n}, I_{n}\right)} \prod_{i=1}^{n-1}\left[r_{1}\left(C_{i}, I_{i}\right) e^{-f_{q_{i}}(L) L^{v}\left|K_{i}\right|}\right] r_{1}\left(C_{n}, I_{n}\right) \sum_{G} \prod_{\mathscr{L} \in G} A(\mathscr{L}),
$$

where $G$ is a graph of lines $\mathscr{L}=\left(I_{i}^{+}, I_{i+1}^{-}\right)$compatible with $J$ in the sense that the 
union of intervals obtained by the above construction is $J$. (By (i)-(iii) above, only the $G$ with every line $\left(I_{i}^{+}, I_{i+1}^{-}\right)$contributes. Hence $\sum \prod A=(-1)^{n+1}$.) If $|J|=1$, then $n=1$ in (4.14), in which case we get

$$
r_{2}(J)=\sum_{I: C(I)=J} r_{1}(J, I)
$$

In both cases we assume $q_{-}\left(I_{1}\right)=q_{-}(J)$ and $q_{+}\left(I_{n}\right)=q_{+}(J)$. In terms of these activities we have

$$
\begin{aligned}
& Z_{\mathrm{res}, 1}(V)=Z_{\mathrm{res}, 2}(V)+Z_{\mathrm{big}, 2}(V), \\
& Z_{\mathrm{res}, 2}(V)=\sum_{n \geqq 0} \frac{1}{n_{J_{1}}, \ldots, J_{n}} \sum_{i=1}^{n}\left[r_{2}\left(J_{i}\right) e^{-f_{q_{i}}(L) L^{v}\left|K_{i}\right|}\right] .
\end{aligned}
$$

Here $K_{i}$ are intervals between components, with boundary condition $q_{i}=q_{+}\left(J_{i}\right)=q_{-}\left(J_{i+1}\right)$. As usual, $Z_{\mathrm{big}, 2}(V)$ is defined as the sum of terms where a single component encircles $T$.

Evidently we have recovered our original form (4.3) for the partition function. The gain here is that any $r_{2}(I)$ with $|I|>1$ is necessarily a two-kink effect. More generally, we can construct $r_{k+1}$ from $r_{k}$ just as $r_{2}$ was constructed from $r_{1}$. As we shall see, $r_{k}(I)$ with $|I|>1$ is at least a $k$-kink effect, decreasing as $\exp \left(-k(\tau-O(1)) L^{v}\right)$ as $k \rightarrow \infty$, only the term with $|I|=1$ is nonzero, and we have the desired product structure.

Proposition 4.1. Suppose that $r_{k}$ satisfies the following bounds:

$$
\begin{gathered}
\sum_{|l| \leqq 4}\left(\frac{1}{L^{v}|I|}\right)^{|l|}\left|\frac{\partial^{l}}{\partial \mu^{l}} r_{k}(I)\right| e^{f(L) L^{v}} \leqq \varepsilon_{1}, \quad|I|=1, \\
\sum_{I \ni 0,|I|>1} \sum_{|l| \leqq 4} e^{\left(\tau^{*}-c_{0}\right)(|I|-1)} e^{f(L) L^{v}|I|}\left(\frac{1}{L^{v}|I|}\right)^{|l|}\left|\frac{\partial^{l}}{\partial \mu^{l}} r_{k}(I)\right| \leqq \varepsilon_{2},
\end{gathered}
$$

for some $c_{0}=O(1)$ (independent of $k$ ). If $\varepsilon_{2} \leqq \varepsilon_{1} \leqq e^{-\left(\tau^{*}-c_{0}\right)}$, then $r_{k+1}$ satisfies the same bounds, but with $\varepsilon_{1} \rightarrow \varepsilon_{1}+O(1) \varepsilon_{2} e^{-\left(\Delta \tau-c_{0}\right)}, \varepsilon_{2} \rightarrow O(1) \varepsilon_{2} \varepsilon_{1} e^{\tau^{*}-\Delta \tau}$.

Remark. By (4.4) and (4.6) $r_{1}$ satisfies the assumptions of the proposition with $\varepsilon_{1}=\varepsilon_{2}=\exp \left(-(\tau-O(1)) L^{\nu}\right)$.

Proof. In the course of the proof, we will encounter derivatives of products of activities and volume factors at several places. All these derivatives will be bounded using the estimate

$$
\sum_{|l| \leqq 4}\left(\frac{1}{L^{v}|I|}\right)^{|l|}\left|\frac{\partial^{l}}{\partial \mu^{l}} \prod_{i=1}^{n} A_{i}(\mu)\right| \leqq 4 ! \prod_{i=1}^{n}\left[\sum_{|l| \leqq 4}\left(\frac{1}{L^{v}|I|}\right)^{|l|}\left|\frac{\partial^{l}}{\partial \mu^{l}} A_{i}(\mu)\right|\right]
$$

which is easily proven using Leibniz's rule.

As a first application we translate our assumption into a bound on $r_{k}(C, I)$. As in (4.9) we obtain

$$
\begin{aligned}
\left\|r_{k}\right\| & \equiv \sum_{\substack{(C, I): \\
I \ni 0,|I|>1}} \sum_{|l| \leqq 4} e^{\left(\tau^{*}-a_{q_{+}} L^{v-}-c_{0}\right)\left|I^{+}\right|} e^{\left(\tau^{*}-a_{q_{-}} L^{\left.v-c_{0}\right) \mid I^{-}-1} e^{f(L) L^{v}}\left(\frac{1}{L^{v}|I|}\right)^{|l|}\left|\frac{\partial^{l}}{\partial \mu^{l}} r_{k}(C, I)\right|\right.} \\
& \leqq \varepsilon_{2} O(1),
\end{aligned}
$$


where we used the fact that the volume factors produced by derivatives are cancelled by the corresponding part of $\left(L^{v}|I|\right)^{-|l|}$. The unit interval estimate is unchanged since $r_{k}(C, C)=r_{k}(C)$.

All that remains is to bound the expansion (4.14), remembering that $\left|\sum \prod A\right|=1$. The case $|J|=1$ is simple, since then (4.5) applies and we have

$$
\begin{aligned}
\sum_{|l| \leqq 4}\left(\frac{1}{L^{v}|J|}\right)^{|l|}\left|\frac{\partial^{l}}{\partial \mu^{l}} r_{k+1}(J)\right| e^{f(L) L^{v}} & \leqq \varepsilon_{1}+e^{-\left(\Delta \tau-c_{0}-O(1)\right)}\left\|r_{k}\right\| \\
& \leqq \varepsilon_{1}+e^{-\left(\Delta \tau-c_{0}-O(1)\right)} \varepsilon_{2} .
\end{aligned}
$$

We used the fact that $\left(L^{v}|J|\right)^{-|l|}=\left(L^{\nu}\right)^{-|l|} \leqq\left(L^{v}|I|\right)^{-|l|} e^{|I| O(1)}$ together with the fact that $|I| \geqq 2$ in $(4.20)$.

The case $|J|>1$ needs some care. We use the fact that there may not be two $r_{k}(C, C)$ 's in a row in (4.14); every pair $\left(C_{i}, I_{i}\right),\left(C_{i+1}, I_{i+1}\right)$ must interact. There must be at least one $\left|I_{i}\right|>1$ and at least two $I_{i}$ 's overall. In order to estimate derivatives, we bound the factor $\left(L^{v}|J|\right)^{-|l|}$ appearing together with the lth derivative on the left-hand side of (4.18) by

$$
\begin{aligned}
\left(\left|\bigcup_{i} I_{i}\right| L^{v}\right)^{-|l|}\left(\frac{\left|\bigcup_{i} I_{i}\right|}{|J|}\right)^{|l|} & \leqq\left(\left|\bigcup_{i} I_{i}\right| L^{v}\right)^{-|l|}\left(1+\left|\bigcup_{i} I_{i}\right|-|J|\right)^{|l|} \\
& \leqq\left(\left|\bigcup_{i} I_{i}\right| L^{v}\right)^{-|l|} \exp \left\{4\left(\left|\bigcup_{i} I_{i}\right|-|J|\right)\right\}
\end{aligned}
$$

and apply (4.19). Derivatives of the term $\prod e^{-f_{q_{i}}(L) L^{\nu}\left|K_{i}\right|}$ produce volume factors $O(1)\left(L^{\nu} \sum\left|K_{i}\right|\right)^{|l|}$ which are cancelled by the corresponding factor $\left(\left|\bigcup_{i} I_{i}\right| L^{\nu}\right)^{-|l|}$, while derivatives of activities will be bounded using the inductive assumption (4.20).

Let us categorize the remaining factors that result from evaluating a term in (4.18) after inserting the expansion (4.14) and the above bounds. Such a term is $O(1)$ times

$$
\begin{aligned}
& e^{\left(\tau^{*}-c_{0}\right)(|J|-1)} e^{f(L) L^{\nu}|J|} \exp \left\{4\left(\left|\bigcup_{i} I_{i}\right|-|J|\right)\right\} \\
& \cdot \prod_{j=1}^{n}\left[\sum_{l}\left(L^{v}\left|I_{j}\right|\right)^{-|l|}\left|\frac{\partial^{l} r_{k}\left(C_{j}, I_{j}\right)}{\partial \mu^{l}}\right|\right]_{j=1}^{n-1} e^{-f_{q_{j}}(L) L^{\nu}\left|K_{j}\right|},
\end{aligned}
$$
where we have bounded $\left|\bigcup_{i} I_{j}\right|^{-|l|}$ by $\left|I_{j}\right|^{-|l|}$. We associate factors with intervals
as follows:

$$
\begin{array}{ll}
\text { intervals } C_{i}: & e^{f(L) L^{v}} \\
\bigcup_{i} I_{i} \backslash J: & \exp \left\{4\left(\left|\bigcup_{i} I_{i}\right|-|J|\right)\right\} \\
K_{i}: & e^{\left(\tau^{*}-c_{0}+f(L) L^{v}-f_{q_{i}}(L) L^{v}\right)\left|K_{i}\right|}=e^{\left(\tau^{*}-a_{q_{i}} L^{v}-c_{0}\right)\left|K_{i}\right|} \\
\text { additionally: } & e^{\left(\tau^{*}-c_{0}\right)(n-1)} .
\end{array}
$$


Now let us fix $I_{1}^{l}, \ldots, I_{m}^{l}$, the long rods with $|I|>1$, and sum over the short ones. Then by (4.17) there results a factor

$$
1+\varepsilon_{1} e^{-\left(\tau^{*}-a L^{\nu}-c_{0}\right)} e^{\left(\tau^{*}-c_{0}\right)}|H| \leqq 1+\varepsilon_{1} e^{a L^{\nu}}|H| \leqq e^{|H|} \leqq e^{\left(\Delta \tau-c_{0}\right) H} e^{-\left(\Delta \tau-c_{0}-1\right)},
$$

for each overlap interval $H$ of the form $I_{j}^{l+} \cap I_{j+1}^{l-}$. (The two factors multiplying $\varepsilon_{1}$ result from increasing $|K|$ by 1 and decreasing $n$ by 1.) On the initial and final sections $I_{1}^{l-}, I_{m}^{l+}$, of length $R$, say, we have a factor

$$
\begin{aligned}
e^{4 R}\left(1+\sum_{h=0}^{R-1} \varepsilon_{1} e^{\left(\tau^{*}-a L^{\nu}-c_{0}\right) h} e^{-4(h+1)} e^{\tau^{*}-c_{0}}\right) & \leqq e^{4 R}\left(1+2 \varepsilon_{1} e^{\left(\tau^{*}-a L^{\nu}-c_{0}-4\right)(R-1)} e^{\tau^{*}-c_{0}-4}\right) \\
& \leqq e^{\left(\tau^{*}-a L^{v}-c_{0}\right) R}\left(1+2 \varepsilon_{1} e^{a L^{\nu}}\right)
\end{aligned}
$$

Here $a=a_{\left.q_{-(} I_{1}^{l}\right)}$, or $a_{q_{+(}\left(I_{m}^{l}\right)}$, and the first exponential in $h$ comes from dropping $K_{1}$ or $K_{n}$, while the second exponential in $h$ comes from increasing $|J|$ by $h+1$. All these factors from summing over short rods can be bounded by the product $\prod_{j} \exp \left[\left(\tau^{*}-a_{q_{+}} L^{v}-c_{0}\right)\left|I_{j}^{l+}\right|+\left(\tau^{*}-q_{q_{-}} L^{v}-c_{0}\right)\left|I_{j}^{l-}\right|\right]$. Each one of these factors is as in the definition of $\left\|r_{k}\right\|$ in (4.20).

To help in performing the sums over $\left(C, I^{l}\right)$, let

$$
\delta=\varepsilon_{1} e^{\tau^{*}-\Delta \tau} \geqq \varepsilon_{1} e^{a_{q} L^{v}},
$$

with the inequality holding for any $q \in Q_{s}(L)$. By assumption, $\delta \leqq e^{-\left(\Delta t-c_{0}\right)}$. We choose one long interval containing 0 (with factor $m$ ) and sum on either side the pairs $\left(C, I^{l}\right)$ such that $I^{l}$ contains an endpoint of the previous interval. For $m \geqq 2$ we get a bound

$$
m(1+2 \delta)^{2}\left(O(1) \varepsilon_{2}\right)^{m} e^{\left(\tau^{*}-c_{0}\right)(m-1)} e^{-\left(\Delta \tau-c_{0}-1\right)(m-1)} \leqq O(1) m \varepsilon_{2}^{2} e^{\left(\tau^{*}-\Delta \tau\right)}(O(1) \delta)^{m-2} .
$$

When $m=1$ we must have at least one factor $\varepsilon_{1}$ from the two tail sums (4.22), so we get a bound $O(1) \varepsilon_{2}\left(4 \delta+4 \delta^{2}\right) \leqq O(1) \varepsilon_{2} \varepsilon_{1} e^{\tau^{*}-\Delta \tau}$. Summing on $m$, we get the desired bound $O(1) \varepsilon_{2} \varepsilon_{1} e^{\tau^{*-}-\Delta \tau}$.

Starting at $\varepsilon_{1}=\varepsilon_{2}=\exp \left(-(\tau-O(1)) L^{v}\right)$, the mapping $\varepsilon_{1} \rightarrow \varepsilon_{1}+O(1) \varepsilon_{2} e^{-\left(\Delta \tau-c_{0}\right)}$, $\varepsilon_{2} \rightarrow O(1) \varepsilon_{2} \varepsilon_{1} e^{\tau^{*}-\Delta \tau}$ converges geometrically, and $\varepsilon_{1}^{(k)}$ is always $\exp \left(-(\tau-O(1)) L^{v}\right)$, while

$$
\varepsilon_{2}^{(k)} \leqq e^{-k(\tau-O(1)) L^{v}}\left(O(1) e^{\tau^{*}-\Delta \tau}\right)^{k-1} .
$$

As a corollary of the proof, we obtain the following estimate on $Z_{\text {big,k }}(V)$, which has an expansion similar to (4.14):

$$
\left|Z_{\text {big }, k}(V)\right| \leqq e^{-\left(\tau^{*}-c_{0}+f(L) L^{v}\right) t} \varepsilon_{2}^{(k)} .
$$

The same estimate holds for $\mu$-derivatives.

As we discard $Z_{\mathrm{big}, k}(V)$, we are finally only interested in

$$
\Gamma_{p q}(L)=\lim _{k \rightarrow \infty} r_{k}\left(C^{p q}\right)
$$

where $|C|=1$. Here the limit is approached exponentially fast. The partition functions $Z_{\text {res, } k}(V)$ have an expansion as in (4.16), but since $r_{k}(J) \rightarrow 0$ as $k \rightarrow \infty$ for $|J|>1$, the limit has the simpler expression

$$
Z_{\mathrm{res}, \infty}=\lim _{k \rightarrow \infty} Z_{\mathrm{res}, k}=\operatorname{Tr}(F+\Gamma)^{t}
$$


Here $F=\operatorname{diag}\left\{\exp \left(-f_{q}(L) L^{\nu}\right)\right\}_{q \in Q_{s}(L)}$. By Proposition 4.1 we have that $\left|\frac{\partial^{k}}{\partial \mu^{k}} \Gamma_{p q}(L)\right| \leqq$ $e^{-(f+\tau-O(1)) L^{v}}$, which completes the proof of (i)-(iii) of Theorem 2.2.

To obtain (iv) and (v) we need to compare $\Gamma$ with the one-kink matrix $\Gamma(1)$ defined in $(2.17 \mathrm{a})$. The difference $\left(r_{2}\left(C^{p q}\right)-\Gamma_{p q}\right) e^{f(L) L^{v}}$ is of order $\varepsilon_{2}^{(2)} e^{-\left(\tau^{*}-c_{0}\right)} \leqq$ $e^{-(2 \tau-O(1)) L^{v}} e^{-\Delta \tau}$. Recall that $r_{2}(C)$ can be expressed in terms of $r_{1}$ using (4.15), (4.8). If we then replace $r_{1}$ with $r_{1}^{(1)}$, the one-kink term, we obtain (2.16). Thus $\left(r_{2}\left(C^{p q}\right)-\Gamma_{p q}^{(1)}\right) e^{f(L) L^{\nu}}$ involves the two-kink term $r_{1}^{(2)}$ only. Summing (4.6) with the appropriate energy factors gives a bound $e^{-(2 \tau-\delta(1)) L^{v}} e^{\tau^{*}-\Delta \tau}$, which thus governs $\left|\Gamma_{p q}-\Gamma_{p q}^{(1)}\right|$ and its derivatives. This proves the remaining statements in Theorem 2.2 .

\section{Surface Tension and Kink-Matrix}

In this section we show that the matrix $\Gamma$ in Theorem 2.2 can be chosen in such a way that

$$
\Gamma_{p q}=\left(1+O\left(e^{-b_{1} \tau L}\right)\right) e^{-\sigma_{p q} L^{v}} e^{-f L^{v}},
$$

if $\mu=\mu^{*}, p \neq q$ and $v+1 \geqq 3$, while

$$
\Gamma_{p q}=O\left(L^{-1 / 2}\right) e^{-\sigma_{p q} L^{v}} e^{-f L^{v}},
$$

if $\mu=\mu^{*}, p \neq q$ and $v+1=2$. Here $\sigma_{p q}$ is the surface tension between $p$ and $q$.

We will prove these results using the surface expansions introduced by Dobrushin [D] (see also [G, BF and HKZ]) to prove these results for the 1-kink matrix $\Gamma_{a b}^{(1)}$, and a stronger version of Theorem 2.2 (iv), see Proposition 5.3 below, to estimate the difference between $\Gamma_{p q}$ and $\Gamma_{p q}^{(1)}$. In order to take into account of the special features of our problem - periodic boundary conditions and the need for bounds on logarithmic derivatives of $\Gamma_{p q}^{(1)}$, see Proposition 5.1 below - we will need some significant extensions of the above-mentioned surface expansions. We will need certain additional assumptions and we have to slightly change the definition of $\Gamma_{p q}^{(1)}$, see Eq. (5.1) below.

In order to formulate the assumptions needed to prove the results of this section, we need some notation. We recall that $\pi$ denotes the orthogonal projection from $V_{\infty}=A \times \mathbb{R}$ onto $A$ and introduce the notion of the height of a point in $V_{\infty}$ by defining the height of $(x, t) \in A \times \mathbb{R}$ as $t$.

Fixing a kink $Y=Y^{p q} \subset V_{\infty}$ we define: a cube $c$ in $Y$ is called simple iff it is the only cube in $Y$ which has the projection $\pi(c)$. A cube $c$ in $Y$ is called excited iff there is a cube $c^{\prime} \neq c$ in $Y$ with dist $\left(\pi(c), \pi\left(c^{\prime}\right)\right)<1$ which isn't simple. A connected component $C$ of the set $Y^{*}$ of excited cubes in $Y$ is called small if $|\pi(c)|<L$ and $v=1$, or if $|\pi(c)|<L / 2$ and $v \geqq 2$. We denote the union of all large components of $Y^{*}$ by $W_{0}$ and the small components of $Y^{*}$ by $W_{1}, \ldots, W_{n} . W_{0}$ will be called the large wall of $Y$ and $W_{1}, \ldots, W_{n}$ are called the small walls of $Y$. Finally, $S \subset Y$ is called a flat piece of $Y$ if it is a connected component of $Y \backslash Y^{*}$. A cube $c$ in a wall $W$ (a flat piece $S$ ) is called a boundary cube of $W$ (of $S$ ), if $\pi(c)$ is connected to $\partial \pi(w)$ (to $\partial \pi(s)$ ). The height of a cube is the height of its center. If $v=1$, the height of a small wall is defined as the height of its right boundary cube minus the height of its left boundary cube, see Fig. 5.1. 

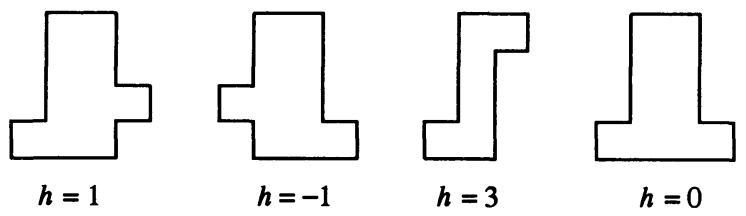

Fig. 5.1. Different walls in $v+1=2$ and their height $h$

Remarks. i) If $W \subset V_{\infty}$ is a union of cubes, it is called a wall if there is a kink $Y$ such that $W$ is a wall of $Y$.

ii) We have defined the notion of a kink $Y$ in such a way that each simple cube $c$ in $Y$ has exactly $2 v$ neighboring cubes (in the sense of sharing a common face) in $Y$. As a consequence, two boundary cubes $c_{W}$ and $c_{S}$ of a wall $W$ and flat piece $S$ have the same height if $\pi\left(c_{W}\right)$ and $\pi\left(c_{S}\right)$ are connected.

iii) If $v=1$, the large wall $W_{0}$ of a kink $Y$ is either empty or $W_{0} \equiv Y$.

In order to redefine $\Gamma^{(1)}$ in such a way that the above-mentioned surface expansions can be applied we now introduce, for $v \geqq 2$ and an arbitrary kink $Y$, the reduced kink $Y_{0}$ by subtracting all small walls from $Y$ and filling the created holes by flat pieces. Note that there is only one way to do this in such that $Y_{0}$ is again a kink due to the above remark ii). We introduce $\widetilde{C}(Y)$ as the unit interval $C\left(Y_{0}\right)$ obtained from $Y_{0}, \tilde{I}_{+}(Y)$ as the part of $I(Y)$ which lies above $\tilde{C}(Y)$ and $\tilde{I}_{-}(Y)$ as the part of $I(Y)$ which lies below $I(Y)$. Our new definition of $\Gamma_{p q}^{(1)}$ is then

$$
\Gamma_{p q}^{(1)}=\sum_{Y} \kappa(Y) e^{-f_{p}(L)\left\{\left|V_{-}(Y)\right|-L^{\nu}\left|\tilde{I}_{-}(Y)\right|\right\}} e^{-f_{q}(L)\left\{\left|V_{+}(Y)\right|-L^{\nu}\left|\tilde{I}_{+}(Y)\right|\right\}}
$$

where the sum goes over all kinks $Y=Y^{p q}$ such that $\tilde{C}(Y)$ is a fixed interval in $\mathbb{R}$, say the interval $\left(-\frac{1}{2}, \frac{1}{2}\right) . V_{ \pm}(Y)$ are as before defined as the upper (lower) part of $(A \times I(Y)) \backslash V(Y)$, cf. Sect. 2. Note that the contributions of the different walls of $Y$ to $\left|V_{ \pm}(Y)\right|-L^{v}\left|\tilde{I}_{ \pm}(Y)\right|$ are additive: if one defines $Y(W)$ as the kink having $W$ as its only wall and $V_{ \pm}(W)=V_{ \pm}(Y(W)), \tilde{I}_{ \pm}(W)=\tilde{I}_{ \pm}(Y(W))$ one easily shows that

$$
\left|V_{ \pm}(Y)\right|-L^{v}\left|\tilde{I}_{ \pm}(Y)\right|=\sum_{i=0}^{n}\left[\left|V_{ \pm}\left(W_{i}\right)-L^{v}\right| \tilde{I}_{ \pm}\left(W_{i}\right) \mid\right]
$$

if $Y$ is a kink with walls $W_{0}, \ldots, W_{n}$ (if $n=0,(5.2 \mathrm{a})$ is obvious; for the induction step $n \rightarrow n+1$ it requires a minute of inspection, the essential fact which is used is the fact that $\tilde{C}(Y)$ is not changed if one adds a small wall $W_{n+1}$ to $Y$ ). while

If $v=1$, it is not possible to define $\tilde{C}(Y)$ in such a way that (5.2a) remains valid:

$$
\begin{aligned}
\Sigma(Y) & \equiv L^{v}\left|\tilde{I}_{+}(Y)\right|+L^{v}\left|\tilde{I}_{-}(Y)\right|-\left|V_{+}(Y)\right|-\left|V_{-}(Y)\right| \\
& =|Y|-|\pi(Y)|+|\operatorname{Int} Y|=\sum\left(\left|W_{i}\right|-\left|\pi\left(W_{i}\right)\right|+\mid \text { Int } W_{i} \mid\right)
\end{aligned}
$$

for all $v$ including $v=1$,

$$
\Delta(Y) \equiv\left(\left|V_{+}(Y)\right|-L^{v}\left|\tilde{I}_{+}(Y)\right|\right)-\left(\left|V_{-}(Y)\right|-L^{v}\left|\tilde{I}_{-}(Y)\right|\right)
$$


cannot be written as a sum of local terms if $v=1$. In order to keep these non-local effects minimal, we define $\widetilde{C}(Y)$ in such a way that $|\Delta(Y)|$ is minimal. Since ||$V_{+}(Y)|-| V_{-}(Y)|| \leqq L^{v}(|I(Y)|-1)$, and

$$
\Delta(Y)=\left|V_{+}(Y)\right|-\left|V_{-}(Y)\right|-m L^{v}
$$

where $m=|I(Y)|-1,|I(Y)|-3, \ldots, 1-|I(Y)|$, depending on the choice of $\tilde{C}(Y)$, we can arrange that $|\Delta(Y)| \leqq L$ if $v=1$ by choosing $\widetilde{C}(Y)$ in such a way that $|\Delta(Y)|$ is minimal.

We now state the assumptions used in this section.

Assumption 5.1 (Factorization). There are functions $\tilde{\sigma}_{a b}(\mu)$ and wall-activities $\tilde{\rho}(W)$ such that

(i) $\rho\left(Y^{a b}\right)=e^{-\left(\tilde{\sigma_{a b}}+\left(e_{a}+e_{b}\right) / 2\right) L^{\nu}} \prod_{i} \tilde{\rho}\left(E_{i}\right)$ for $a \neq b$ and each kink $Y^{a b}$ with walls
$W_{0}, \ldots, W_{n}$. (ii) $\tilde{\rho}(W)$ is translation-invariant.

Assumption 5.2 (Suppression of Excitations). For an arbitrary wall $W$,

$$
|\tilde{\rho}(W)| \leqq e^{-\left(\tau+e_{0}\right)(|W|-|\pi(W)|)} .
$$

Assumption 5.3 (Bounds on Derivatives). There are constants $C_{k}, 1 \leqq|k| \leqq 4$, not depending on $\tau$ and $L$, such that

$$
\left|\frac{d^{k}}{d \mu^{k}} \tilde{\sigma}_{a b}\right| \leqq C_{k}
$$

and

$$
\left|\frac{d^{k}}{d \mu^{k}} \tilde{\rho}(W)\right| \leqq C_{k} e^{-\left(\tau+e_{0}\right)(|W|-|\pi(W)|)}
$$

Assumption 5.4 (Suppression of 2-Kink Terms Relative to 1-Kink Terms). Let $a, b, c$ be distinct elements of $Q$. Then,

$$
\tilde{\sigma}_{a c}+\tilde{\sigma}_{c b} \geqq \tilde{\sigma}_{a b}+\tau-O(1) .
$$

Assumption 5.5 (Additional Assumptions for $v=1$ ).

(i) The wall activities $\tilde{\rho}(W)$ and the activities $\rho(Y)$ of all ordinary contours $Y$ are invariant under reflections with respect to the vertical lattice planes $x_{0} \times \mathbb{R}, x_{0} \in A$.

(ii) $\tilde{\rho}(W) \geqq 0$ for all small walls $W$.

(iii) There exists at least one small wall $W$ with height $h \neq 0$ such that $\tilde{\rho}(W)>0$.

Before stating the theorems proven in this section we want to comment on the above assumptions. Assumptions 5.1 requires that the kink activity $\rho$ is the product of the flat kink activity $\exp \left(-\left(\tilde{\sigma}_{a b}+\left(e_{a}+e_{b}\right) / 2\right) L^{\nu}\right)$ and certain translation invariant activities $\tilde{\rho}\left(W_{i}\right)$ for the pieces describing the deviations from the flat kink. It is typical of models with finite-range interactions. Note that Assumption 5.1 is only compatible with assumption (2.1) of Sect. 2 if $\tilde{\sigma}_{a b} \geqq \tau$.

In contrast to Assumption 5.1, Assumptions 5.2 through 5.4 significantly restrict the class of models for which the methods of this section apply if there are more than two states, $N>2$. Assumption 5.4 fails if it is more favorable to pass from phase $a$ to $b$ via phase $c$ than directly from $a$ to $b$. For example, in a model with 
spins $s_{x}=0, \pm 1$ and nearest neighbor interaction $J\left|s_{x}-s_{y}\right|$, we have $\tilde{\sigma}_{0+}=\tilde{\sigma}_{0-}=J$ and $\tilde{\sigma}_{+-}=2 J$. Since a wall $W$ may be a splitting of the kink $Y^{a b}$ into two surfaces involving energies $\tilde{\sigma}_{a c}$ and $\tilde{\sigma}_{c b}$, Assumptions 5.2 and 5.3 will, in general, also fail if 5.4 fails for these models. Note that the methods of this section can still be applied to a certain subclass of kinks, for example if $a$ and $b$ are such that $\tilde{\sigma}_{a b} \leqq \min _{c, d} \tilde{\sigma}_{c d}+O(1)$. We do not investigate this case any further here.

On the other hand, Assumptions 5.2 and 5.3 should, in general, be valid if the interaction range is finite and Assumption 5.4 is fulfilled.

Proposition 5.1. Assume that Assumptions 5.1 through 5.3 are valid, that $v+1 \geqq 3$ and that $p \neq q$. Then there is a constant $b_{2}>0$ and $a C^{4}$-function $\sigma_{p q}(\mu)$ such that

(i) $\sigma_{p q}(\mu)$ is the surface tension between $p$ and $q$ if $p$ and $q$ are stable, i.e., if $f_{p}(\mu)=f_{q}(\mu)=f(\mu)$.

(ii) For $|k| \leqq 4$,

$$
\left|\frac{d^{k}}{d \mu^{k}}\left(\sigma_{p q}-\tilde{\sigma}_{p q}\right)\right| \leqq O\left(e^{-b_{2} \tau}\right) .
$$

(iii) If $a_{q} L^{v}$ and $a_{p} L^{v}$ are smaller than $7 \tau / 8$, and if $|k| \leqq 4$,

$$
\left|\frac{d^{k}}{d \mu^{k}}\left[\Gamma_{p q}^{(1)}-e^{-\left(\sigma_{p q}+\left(f_{q}+f_{p}\right) / 2\right) L^{v}}\right]\right| \leqq e^{-\left(\sigma_{p q}+\left(f_{q}+f_{p}\right) / 2\right) L^{v}} e^{-b_{2} \tau L} .
$$

Proposition 5.2. Assume that $v+1=2$, and that Assumptions 5.1, 5.2, 5.3 and 5.5 are valid. Let $p \neq q$, and define $\Delta f=\left(f_{q}-f_{p}\right) / 2$ and $a(\mu)=\max \left\{a_{p}(\mu), a_{q}(\mu)\right\}$. Then the following statements are true:

(i) If $p$ and $q$ are stable (i.e., $f_{p}(\mu)=f_{q}(\mu)=f(\mu)$ ), the limit

$$
\sigma_{p q}=f(\mu)-\lim _{L \rightarrow \infty} \frac{1}{L} \log \Gamma_{p q}^{(1)}(L, \mu)
$$

exists and

$$
\left|\sigma_{p q}-\tilde{\sigma}_{p q}\right| \leqq O\left(e^{-b_{2} \tau}\right)
$$

(ii) If $a(\mu) L \leqq O(1)$,

$$
e^{-\left(|\Delta f|-O\left(e^{-b_{2} \tau}\right)\right) L} \leqq \Gamma_{p q}^{(1)}(L, \mu) e^{\left(f(\mu)+\tilde{\sigma}_{p q}(\mu)\right) L} \leqq e^{\left(|\Delta f|+O\left(e^{-b_{2} \tau}\right)\right) L} .
$$

(iii) If $a(\mu) L \leqq O(1)$,

$$
\left|\frac{d}{d \mu_{i}} \Gamma_{p q}^{(1)}(L, \mu)\right| \leqq O(L) \Gamma_{p q}^{(1)}(L, \mu)\left\{1+|\Delta f| r^{L O\left(e^{-b_{1} \tau}\right)}\right\} .
$$

(iv) There is a constant $C_{p q}>0$ such that

$$
\Gamma_{p q}^{(1)}(L, \mu)=C_{p q} L^{-1 / 2} e^{-\left(f+\sigma_{p q}\right) L}\left(1+O\left(L^{-1}\right)\right)
$$

provided $p$ and $q$ are stable (i.e., $a(\mu)=0$ ) and $L$ is large enough.

Assumption 5.5(iii) is not needed to prove (i) through (iii), and Assumption 5.5(ii) is not needed to prove (i). 
Remark. If $v=1$ and Assumption 5.5(iii) is not valid, i.e, $\tilde{\rho}(W)=0$ for all walls with height $h \neq 0$,

$$
\Gamma_{p q}^{(1)}(\mu)=e^{-\left(f+\sigma_{p q}\right) L}\left(1+O\left(e^{-\tau b_{2} L}\right)\right)
$$

provided $p$ and $q$ are stable (i.e., $a(\mu)=0$ ).

Proposition 5.3. Assume that Assumptions 5.1 through 5.4 are valid for all $a \neq b$. Then the bounds (iii) and (iv) of Theorem 2.2 can be sharpened to

$$
\left|\frac{d^{k}}{d \mu^{k}} \Gamma_{a b}(L)\right| \leqq e^{-\left(f+\tilde{\sigma}_{a b}-O(1)\right) L^{v}},
$$

and

$$
\left|\frac{d^{k}}{d \mu^{k}}\left(\Gamma_{a b}(L)-\Gamma_{a b}^{(1)}(L)\right)\right| \leqq e^{-\left(f+\tilde{\sigma}_{a b}-O(1)\right) L^{v}} e^{-\tau\left(L^{\nu}-1\right)},
$$

provided $a, b \in Q_{s}(L)$ and $a \neq b$. Assumption 5.3 is not needed to prove (5.6) for $k=0$, and Assumption 5.4 is not needed if $N \leqq 2$.

Proof of Proposition 5.3. By the Assumption 5.1 through 5.3,

$$
\left|\frac{d^{k}}{d \mu^{k}} \rho\left(Y^{a b}\right)\right| e^{e_{0}\left|Y^{a b}\right|} \leqq e^{-\left(\tilde{\sigma}_{a b}-O(1)\right) L^{v}} e^{-(\tau-O(1))\left(\left|Y^{a b}\right|-L^{v}\right)},
$$

from which we conclude that

$$
\left|\frac{d^{k}}{d \mu^{k}} \kappa\left(Y^{a b}\right)\right| \leqq e^{-\left(\tilde{\sigma}_{a b}-O(1)\right) L^{v}} e^{-(\tau-O(1))\left(\left|Y^{a b}\right|-L^{\nu}\right)} e^{-f(L)|V(Y)|},
$$

where $\kappa(\cdot)$ is the activity introduced in Sect. 3. In order to treat the case where $a=b$ at the same time as the case $a \neq b$ we set $\tilde{\sigma}_{a a}=\tau$ for the purpose of this proof (note that $\left(3.20^{\prime}\right)$ is just (3.20) if $a=b$ with the notation). As a consequence of $\left(3.20^{\prime}\right)$ we may improve the 1-kink bound (4.4) of Sect. 4 to

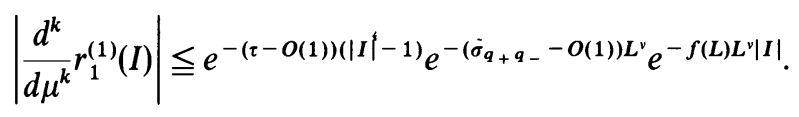

In order to improve the bound (4.6) or $r_{1}^{(2)}(I)$, we use $\left(3.20^{\prime}\right)$ together with Assumption 5.4. We obtain

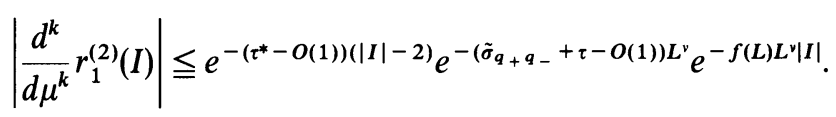

Using (4.4') and (4.6'), the proof of (5.6) is the same as that of the bounds (iii) and (iv) of Theorem 2.2. The fact that Assumption 5.3 is not needed to prove (5.6) for $k=0$ is obvious.

In order to prove Propositions 5.1 and 5.2 we need some facts about the geometry of a general kink $Y$. We denote by $\varphi_{s}$ the translation $\varphi_{s}:(x, t) \mapsto(x, t+s)$ and call a general translation $\varphi=\varphi_{s}, s \in \mathbb{Z}$ a vertical translation. For a set $C$ of cubes in $V_{\infty}$ we use [C] to denote the equivalence class

$$
[C]=\left\{C^{\prime} \mid C^{\prime}=\varphi_{s}(C), s \in \mathbb{Z}\right\},
$$




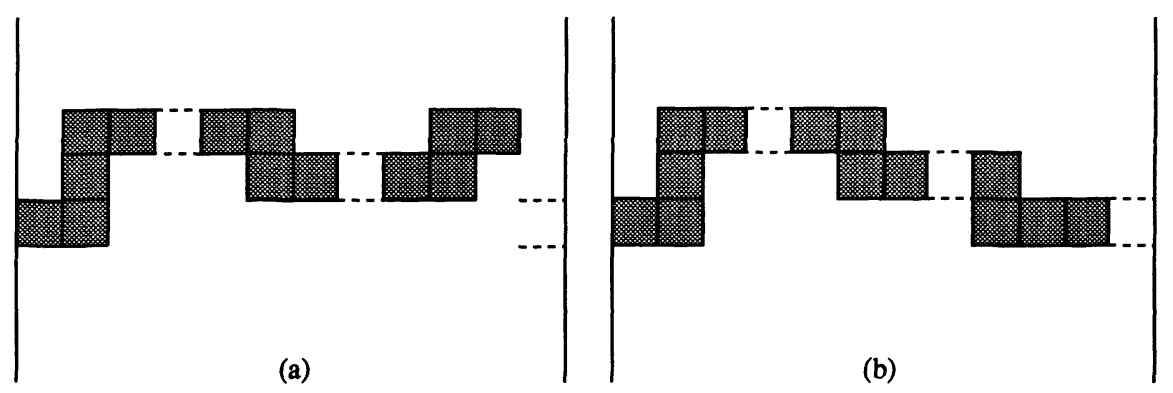

Fig. 5.2 a, b. A set of mutually compatible walls not corresponding to a kink (a) and a set of mutually compatible walls corresponding to a kink in $v+1=2$ dimensions

under vertical translations. If $Y$ is a kink with walls $W_{0}, \ldots, W_{n}$, we call $\left[W_{0}\right], \ldots,\left[W_{n}\right]$ the "floating" walls of $Y$.

Given two walls $W, W^{\prime}$, we say that $[W]$ and $\left[W^{\prime}\right]$ are compatible, if $\pi(W)$ and $\pi\left(W^{\prime}\right)$ do not share a $(v-1)$-dimensional cube. With this definition, the floating walls $\left[W_{0}\right], \ldots,\left[W_{n}\right]$ of any given kink $Y$ are mutually compatible.

Lemma 5.4. Let $v \geqq 2$ and $\left\{\left[W_{0}\right], \ldots,\left[W_{n}\right]\right\}$ be a set of mutually compatible floating walls, where $W_{0}$ is large and $W_{1}, \ldots, W_{n}$ are small. Then there is exactly one kink $Y$ with $\widetilde{C}(Y)=\left(-\frac{1}{2}, \frac{1}{2}\right)$ which has $\left[W_{0}\right], \ldots,\left[W_{n}\right]$ as its floating walls.

Lemma 5.5. Let $v=1$ and let $\left\{\left[W_{1}\right], \ldots,\left[W_{n}\right]\right\}$ be a set of mutually compatible, small floating walls whose heights add up to zero. Then there is exactly one kink $Y$ with $C(Y)=\left(-\frac{1}{2}, \frac{1}{2}\right)$ which has $\left[W_{0}\right], \ldots,\left[W_{n}\right]$ as its floating walls.

Proof of Lemmas 5.4 and 5.5. These lemmas are an immediate consequence of Remark (ii) before Assumption 5.1; the corresponding geometric conditions have been discussed at length in [D], and are not repeated here. Note that the conditions $C(Y)=\left(-\frac{1}{2}, \frac{1}{2}\right)$ and $\widetilde{C}(Y)=\left(-\frac{1}{2}, \frac{1}{2}\right)$ fix the global horizontal translation which is fixed in [D] by fixing the boundary of the interface studied there. See Fig. 5.2 for an illustration of Lemma 5.5.

Proof of Proposition 5.1. We will prove this proposition by rewriting (5.1) as the partition function of a polymer gas in $A$. Neglecting, for a moment, the factor

$$
e^{g_{p}(\partial-Y)} e^{g_{q}(\partial+Y)}
$$

in the activity $\kappa(Y)=\kappa\left(Y^{p q}\right)$, see Eq. (3.18), we rewrite (5.1) as

$$
\begin{aligned}
\Gamma_{p q}^{(1)} & \left.\approx \sum_{Y} \rho(Y) \prod_{m} Z_{m}\left(\operatorname{Int}_{m} Y\right) e^{-f_{p}(L)\left\{\left|V-1-L^{\nu}\right| \tilde{I}-\mid\right.}\right\} e^{-f_{q}(L)\left\{\left|V+1-L^{\nu}\right| \tilde{I}+\mid\right\}} \\
& =e^{-\left(\tilde{\sigma}_{a b}+(1 / 2)\left(e_{a}+e_{b}\right)\right) L^{\nu}} \sum_{Y} \prod_{i=0}^{n} \tilde{K}\left(W_{i}\right)
\end{aligned}
$$

where $W_{0}, \ldots, W_{n}$ are the walls of $Y$. We used (5.2a) and Assumption 5.1 and defined $\tilde{K}(W)=\tilde{\rho}(W) \prod_{m} Z_{m}\left(\operatorname{Int}_{m} W\right) e^{-f_{p}(L)\left\{\left|V_{-}(W)\right|-L^{\nu}\left|\tilde{I}_{-}(W)\right|\right\}} e^{-f_{q}(L)\left\{\left|V_{+}(W)\right|-L^{\nu}\left|\tilde{I}_{+}(W)\right|\right\}}$. 
In order to define $\sigma_{a b}(\mu)$ we will also need certain truncated activities,

$$
\begin{aligned}
\tilde{K}^{\prime}(W)= & \tilde{\rho}(W) \prod_{m} Z_{m}\left(\operatorname{Int}_{m} W\right) e^{-f_{p}\left\{|V-(W)|-L^{v}\left|\tilde{I}_{-}(W)\right|\right\}} e^{-f_{q}\left\{\left|V_{+}(W)\right|-L^{\nu}\left|\tilde{I}_{+}(W)\right|\right\}} \\
& \cdot \chi\left(\frac{7 \tau}{8}-a_{p}|S(W)|\right) \chi\left(\frac{7 \tau}{8}-a_{q}|S(W)|\right)
\end{aligned}
$$

where $S(W)$ is the union of $\pi(W)$ and its interior, while $\chi$ is a smoothed characteristic function $\chi \in C^{4}$, with $0 \leqq \chi \leqq 1, ~ \chi(x)=0$ if $x \leqq-1, \chi(x)=1$ if $x \geqq 0$ and $\left|d^{k} \chi / d x^{k}\right| \leqq O(1)$ for $1 \leqq k \leqq 4$. We will need the following lemma which will be proven below.

Lemma 5.6. Let $p \neq q$ and $|k| \leqq 4$. Then,

$$
\left|\frac{d^{k}}{d \mu^{k}} \tilde{K}^{\prime}(W)\right| \leqq e^{-(\tau / 8-O(1))(|W|-|\pi(W)|)},
$$

for all small walls $W$.

$$
\left|\frac{d^{k}}{d \mu^{k}} \tilde{K}(W)\right| \leqq e^{-(\tau / 8-O(1))(|W|-|\pi(W)|)},
$$

for all walls $W$, provided

$$
a_{p} L^{v} \leqq \frac{7}{8} \tau \text { and } a_{q} L^{v} \leqq \frac{7}{8} \tau
$$

$$
\left|\frac{d^{k}}{d \mu^{k}}\left[\tilde{K}(W)-\tilde{K}^{\prime}(W)\right]\right| \leqq e^{-(\tau / 8-O(1))(|W|-|\pi(W)|)} O\left(e^{-b_{1} \tau L}\right),
$$

provided $W$ is a small wall and the condition (5.11) is fulfilled.

Up to the interactions between different walls coming from the neglected term (5.7), we have rewritten $\Gamma_{p q}^{(1)}$ as a dilute polymer gas with hard-core interaction. We follow [BF], see also [HKZ], to take into account the additional interaction coming from (5.7). In a first step we express $g_{m}\left(\partial_{ \pm} Y\right)$ as a sum over sets $X$ intersecting $\partial_{ \pm} Y$,

$$
g_{m}\left(\partial_{ \pm} Y\right)=\sum_{X} k_{m}(X)
$$

where $k_{m}(X)$ obeys a bound

$$
\left|\frac{d^{k}}{d \mu^{k}} k_{m}(X)\right| \leqq e^{-(\tau / 8-o(1))|X|},
$$

if the condition (5.11) is fulfilled (cf. Sect. 3). Expanding $\exp \left(k_{m}(X)\right)$ as $1+\tilde{k}_{m}(X)$, where $\tilde{k}_{m}(X)$ obeys again a bound of the form (5.13), we may rewrite $\Gamma_{p q}^{(1)}$ as a sum of terms of the form

$$
e^{-\left(\tilde{\sigma}_{a b}+\left(e_{a}+e_{b}\right) / 2\right) L^{v}} \prod_{i=0}^{n} \tilde{K}\left(W_{i}\right) \prod_{j=1}^{n^{\prime}} \tilde{k}_{m_{j}}\left(X_{j}\right)
$$

where $m_{j}=p$ or $q$. Considering the union of $Y$ with $X_{1}, \ldots, X_{n^{\prime}}$ as a new kink and 
decomposing this new kink again into walls and flat pieces, one finally obtains that $\Gamma_{p q}^{(1)}$ may be written as

$$
\Gamma_{p q}^{(1)}=e^{-\left(\tilde{\sigma}_{p q}+\left(e_{p}+e_{q}\right) / 2\right) L^{\nu}} \sum_{Y} \prod_{i=0}^{n} \tilde{K}\left(W_{i}\right),
$$

where $K(W)$ obeys again a bound of the form (ii) (see Lemma 5.6 above). If we define $K^{\prime}(W)$ by substituting $\widetilde{K}^{\prime}\left(W_{i}\right)$ and $\tilde{k}_{m_{i}}^{\prime}\left(X_{i}\right)$ into $(5.14)$, where $\tilde{k}_{m}^{\prime}\left(X_{i}\right)$ is obtained from the activity $k_{m}^{\prime}(X)$ introduced at the end of Sect. 3 by expanding

$$
e^{k_{m}^{\prime}(X)}=1+\tilde{k}_{m}^{\prime}(X)
$$

the other statements of Lemma 5.6 remain true as well. We summarize these facts in the following:

Lemma 5.7. There are wall activities $K(W)$ and $K^{\prime}(W)$ such that (i) the statements of Lemma 5.6 remain true for $K$ and $K^{\prime}$ and (ii)

$$
\Gamma_{p q}^{(1)}=e^{-\left(\tilde{\sigma}_{p q}+\left(e_{p}+e_{q}\right) / 2\right) L^{\nu}} \sum_{Y} \prod_{i} K\left(W_{i}\right)
$$

provided

$$
a_{p} L^{v} \leqq \frac{7}{8} \tau \quad \text { and } \quad a_{q} L^{v} \leqq \frac{7}{8} \tau
$$

At this point the proof of Proposition 5.1 is straightforward. We introduce "partition functions"

$$
\begin{aligned}
Z(A) & =\sum_{\left\{\left[W_{0}\right], \ldots,\left[W_{n}\right]\right\}} \prod_{i} K\left(W_{i}\right), \\
Z_{\text {small }}(A) & =\sum_{\left\{\left[W_{1}\right], \ldots,\left[W_{n}\right]\right\}} \prod_{i} K\left(W_{i}\right),
\end{aligned}
$$

and

$$
Z^{\prime}(A)=\sum_{\left\{\left[W_{1}\right], \ldots,\left[W_{n}\right]\right\}} \prod_{i} K^{\prime}\left(W_{i}\right)
$$

where the sums go over sets of mutually compatible floating walls, $\left[W_{0}\right]$ (if present) is large, and $\left[W_{1}\right], \ldots,\left[W_{n}\right]$ are small. Due to Lemmas 5.6 and $5.7, Z^{\prime}(A)$ can be analyzed by a convergent cluster expansion. We conclude that the limit

$$
h_{p q}(\mu)=-\lim _{A \rightarrow \mathbb{R}^{v}} \frac{1}{|A|} \log Z^{\prime}(A),
$$

exists, that it obeys the bounds

$$
\left|\frac{d^{k}}{d \mu^{k}} h_{p q}(\mu)\right| \leqq e^{-(\tau / 8-o(1))}
$$

and that

$$
\left|\frac{d^{k}}{d \mu^{k}}\left[\log Z^{\prime}(A)+L^{\nu} h_{p q}(\mu)\right]\right| \leqq e^{-(\tau / 8-O(1)) L},
$$

where we used the fact that the corrections to the infinite volume behavior are 
exponentially small for periodic partition functions. Putting

$$
\sigma_{p q}(\mu)=\tilde{\sigma}_{p q}(\mu)+\frac{1}{2}\left[e_{p}(\mu)+e_{q}(\mu)-f_{p}(\mu)-f_{q}(\mu)\right]+h_{p q}(\mu),
$$

we have defined a $C^{4}$-function which obeys Proposition 5.1(ii).

To see that $\sigma_{p q}$ is the surface tension if $a_{p}=a_{q}=0$, we note that

$$
\left|\tilde{I}_{+}(W)\right| L^{v}+\left|\tilde{I}_{-}(W)\right| L^{v}-\left|V_{+}(W)\right|-\left|V_{-}(W)\right|=|W|+|\operatorname{Int} W|-|\pi(W)|,
$$

from which we conclude that

$$
\tilde{K}^{\prime}(W)=\tilde{\rho}(W) e^{f(|W|-|\pi(W)|)} \prod_{m}\left[Z_{m}\left(\operatorname{Int}_{m} W\right) e^{f|\operatorname{lnt} m|}\right],
$$

if $a_{p}=a_{q}=0$. But this is just the weight of a wall in the usual surface expansion for the surface tension.

We are left with the proof of the bound (iii). By Lemma 5.6(iii) and the fact that both $Z^{\prime}(A)$ and $Z_{\text {small }}(A)$ may be analyzed by a convergent cluster expansion,

$$
\left|\frac{d^{k}}{d \mu^{k}}\left[\log Z^{\prime}(A)-\log Z_{\text {small }}(A)\right]\right| \leqq|A| O\left(e^{-b_{1} \tau L}\right) .
$$

Combined with the bound (5.16b) this implies that

$$
\left|\frac{d^{k}}{d \mu^{k}}\left[Z_{\text {small }}(A)-e^{-L^{v} h_{p q}}\right]\right| \leqq-e^{-L^{v} h_{p q}} O\left(L^{v} e^{-b_{1} \tau L}\right),
$$

which leaves us with the analysis of the difference

$$
Z_{\text {big }}(A)=Z(A)-Z_{\text {small }}(A)=\sum_{\left\{\left[W_{0}\right], \ldots,\left[W_{n}\right]\right\}}^{\prime} \prod_{i} K\left(W_{i}\right),
$$

where the sum goes over sets $\left\{\left[W_{0}\right], \ldots,\left[W_{n}\right]\right\}$, where $\left[W_{0}\right]$ is not empty. Resumming the small walls $\left[W_{1}\right], \ldots,\left[W_{n}\right]$ and using the fact that this resummation can be controlled by a convergent cluster expansion we bound

$$
\begin{aligned}
\left|Z_{\text {big }}(A)\right| & \leqq \sum_{\left[W_{0}\right]}^{\prime} K\left(W_{0}\right) e^{-\left|A \backslash \pi\left(W_{0}\right)\right| h_{p q}+\left|\partial W_{0}\right|} \\
& \leqq e^{-|A| h_{p q}} \sum_{\left[W_{0}\right]}^{\prime} e^{-(\tau / 8-O(1))\left[\left|W_{0}\right|-\left|\pi\left(W_{0}\right)\right|\right]},
\end{aligned}
$$

where we have used the fact that $\left|\pi\left(W_{0}\right)\right| \leqq O(1)\left[\left|W_{0}\right|-\left|\pi\left(W_{0}\right)\right|\right]$. Using the fact that each component of $\left[W_{0}\right]$ is larger than $L / 2$, we immediately obtain the bound

$$
\left|Z_{\mathrm{big}}(A)\right| \leqq e^{-|A| h_{p q}} O\left(e^{-b_{2} \tau L}\right)
$$

where $b_{2}>0$ is a constant. The bounds on derivatives are similar. This completes the Proof of Proposition 5.1.

Proof of Lemma 5.6. We start with the proof of (i). We assume without loss of generality that

$$
a_{p}|S(W)| \leqq \frac{7}{8} \tau+1 \text { and } a_{q}|S(W)| \leqq \frac{7}{8} \tau+1,
$$

because $\tilde{K}^{\prime}(W)$ is zero otherwise. Using Lemma A.2(i) to bound $Z_{m}\left(\operatorname{Int}_{m} W\right)$, the fact that $\left|e_{0}-f\right| \leqq O(1)$ to replace $e_{0}$ in the bound on $\tilde{\rho}(W)$ by $f-O(1)$, and 
Eq. (5.18) and the bounds

$$
\begin{gathered}
{\left[L^{\nu}\left|\tilde{I}_{ \pm}(W)\right|-\left|V_{ \pm}(W)\right|\right] \leqq\left|\tilde{I}_{ \pm}(W)\right||S(W)|,} \\
\left(\left|\tilde{I}_{-}(W)\right|+\left|\tilde{I}_{+}(W)\right|\right)|S(W)| \max \left\{a_{p}, a_{q}\right\} \leqq\left(\frac{7}{8} \tau+1\right)(|W|-|\pi(W)|)
\end{gathered}
$$

to estimate the volume factors in $\tilde{K}^{\prime}(W)$ we obtain the bound

$$
\left|\tilde{K}^{\prime}(W)\right| \leqq e^{-(\tau / 8-O(1))(|W|-|\pi(W)|)} e^{O(1)|\partial W|} \leqq e^{-(\tau / 8-O(1))(|W|-|\pi(W)|)} .
$$

Derivatives produce harmless factors $O(1)|| V_{ \pm}(W)\left|-L^{v}\right| \tilde{I}_{-}(W)||, O(1)|\operatorname{Int} W|$ and $O(1)|S(W)|$ which may be absorbed into a term $e^{O(1)|W|} \leqq e^{O(1)(|W|-|\pi(W)|)}$.

The proof of (ii) is essentially the same as the proof of (i) $\left(f_{m}(L)-f_{m}\right.$ is $O\left(e^{-b_{1} \tau L}\right)$ and therefore harmless). To prove (iii) we note that the condition (5.11) implies that the characteristic functions in (5.10) are 1. Bounding

$$
\left|e^{-f_{m}(L)\left\{\left|V_{ \pm}(W)\right|-L^{\prime}\left|\tilde{I}_{ \pm}(W)\right|\right\}}-e^{-f_{m}\left\{V_{ \pm}(W)\left|-L^{\prime}\right| \tilde{I}_{ \pm}(W) \mid\right\}}\right|
$$

by

$$
e^{-f_{m\{}\left\{V_{ \pm}(W)\left|-L^{y}\right| \tilde{I}_{ \pm}(W) \mid\right\}}\left|e^{|S(W)|(|W|-|\pi(W)|) O\left(e^{\left.-\tau b_{1} L\right)}\right.}-1\right|,
$$

and estimating

$$
\begin{aligned}
\left|e^{|S(W)|(|W|-|\pi(W)|) O\left(e^{-\tau b}, L\right)}-1\right| & \leqq e^{L^{v}(|W|-|S(W)|) O\left(e^{-\tau b} b_{1} L\right.}-1 \\
& \leqq e^{(|W|-|\pi(W)|) O(1)} O\left(L^{v} e^{-\tau b_{1} L}\right),
\end{aligned}
$$

we may prove (iii) in the same way as (i) and (ii).

Proof of Proposition 5.2. In a first step we approximate $\Gamma_{p q}^{(1)}$ by

$$
\tilde{\Gamma}_{p q}^{(1)}=\sum_{Y}^{\prime} \rho(Y) \prod_{m} Z_{m}\left(\operatorname{Int}_{m} Y\right) e^{g_{p}(\partial-Y)+g_{q}\left(\partial_{+} Y\right)} e^{-f_{p}\left\{|V-|-L^{\eta}|I-|\right\}} e^{-f_{q}\left\{\left|V_{+}\right|-L^{\eta}|I+|\right\}}
$$

where the sum $\sum^{\prime}$ goes over kinks $Y$ having only small walls. The resulting error may easily be bounded; using the fact that $\left|W_{0}\right|-\left|\pi\left(W_{0}\right)\right| \geqq O(L)$ for a large wall $W$, together with the bound $\left|d^{k}\left(f_{m}(L)-f\right) / d \mu^{k}\right| \leqq O\left(e^{-b_{1} \tau L}\right)$, one finds that

$$
\left|\frac{d^{k}}{d \mu^{k}}\left[\tilde{\Gamma}_{p q}^{(1)}-\Gamma_{p q}^{(1)}\right]\right| \leqq e^{-\left(f+\tilde{\sigma}_{p q}-O(1)\right) L} O\left(e^{-b_{1} \tau L}\right),
$$

provided $|k| \leqq 4$ and

$$
a(\mu) L \leqq \frac{7 \tau}{8}
$$

Introducing

$$
\bar{f}=\frac{f_{q}+f_{p}}{2} \text { and } \Delta f=\frac{f_{q}-f_{p}}{2},
$$

and using the relation $(5.2 \mathrm{~b})$ we then rewrite

$$
\begin{aligned}
\tilde{\Gamma}_{p q}^{(1)} & =\sum_{Y}^{\prime} \rho(Y) \prod_{m} Z_{m}\left(\operatorname{Int}_{m} Y\right) e^{\{|V(Y)|-L\} \bar{f}} e^{g_{p}(\partial-Y)+g_{q}(\partial+Y)} e^{-(\Delta f) \Delta(Y)} \\
& =e^{-\left(\tilde{\sigma}_{p q}+\left(e_{p}+e_{q}\right) / 2\right) L} \tilde{Z}(L),
\end{aligned}
$$


where $\Delta(Y)$ is the quantity introduced in (5.3) (recall that $|\Delta(Y)| \leqq L)$ and

$$
\tilde{Z}(L)=\sum_{Y}^{\prime} e^{g_{p}(\partial-Y)+g_{q}\left(\partial_{+} Y\right)} e^{-(\Delta f) \Delta(Y)} \prod_{i} \tilde{K}\left(W_{i}\right) .
$$

Here

Defining

$$
\tilde{K}(W)=\tilde{\rho}(W) e^{\bar{f}\{|W|-|\pi(W)|\}} \prod_{m} e^{\bar{f}|\mathbf{I n t} m W|} Z_{m}\left(\operatorname{Int}_{m} W\right) .
$$

$$
\tilde{K}^{\prime}(W)=\tilde{K}(W) \chi\left(\frac{7 \tau}{8}-a_{p}|\pi(W)|\right) \chi\left(\frac{7 \tau}{8}-a_{q}|\pi(W)|\right)
$$

we may proceed as in the proof of Proposition 5.1 to construct activities $K(W)$, $K^{\prime}(W)$ such that

$$
\tilde{Z}(L)=\sum_{Y}^{\prime} e^{-(\Delta f) \Delta(Y)} \prod_{i} K\left(W_{i}\right),
$$

$K$ and $K^{\prime}$ obey the bounds of Lemma 5.6(i) and (ii) and $K(W)=K^{\prime}(W)$ if (5.21) is true.

Next we introduce

$$
\begin{aligned}
& Z(L)=\sum_{Y}^{\prime} \prod_{i} K\left(W_{i}\right)=\sum_{\left\{\left[W_{0}\right], \ldots,\left[W_{n}\right]\right\}_{0}} \prod_{i} K\left(W_{i}\right), \\
& Z^{\prime}(L)=\sum_{Y}^{\prime} \prod_{i} K^{\prime}\left(W_{i}\right)=\sum_{\left\{\left[W_{0}\right], \ldots,\left[W_{n}\right]\right\}_{0}} \prod_{i} K^{\prime}\left(W_{i}\right),
\end{aligned}
$$

where the last sum in both equations goes over sets of walls satisfying the constraint that their heights add up to zero. The error between $Z(L)$ and $\tilde{Z}(L)$ may then be bounded by

$$
\begin{gathered}
Z(L) e^{-L|\Delta f|} \leqq \tilde{Z}(L) \leqq Z(L) e^{L|\Delta f|} \\
\left|\frac{d}{d \mu_{i}}(Z(L)-\tilde{Z}(L))\right| \leqq O(L) \tilde{Z}(L)+L|\Delta f| e^{L\left(|\Delta f|+O\left(e^{\left.\left.-\tau b_{1}\right)\right)}\right.\right.}
\end{gathered}
$$

provided (5.21) is valid.

While (5.25a) is an immediate consequence of the bound $|\Delta(Y)| \leqq L$ and the fact that the activities $\widetilde{K}$ in (5.24a) are positive, the bound (5.25b) requires some comments. Obviously

$$
\frac{d}{d \mu_{i}} \tilde{Z}(L)=-\frac{d(\Delta f)}{d \mu_{i}} \tilde{B}_{1}+\tilde{B}_{2}
$$

where

$$
\begin{aligned}
\tilde{B}_{1} & =\sum_{Y}^{\prime} \Delta(Y) e^{-(\Delta f) \Delta(Y)} \prod_{k} K\left(W_{k}\right) \\
& =\sum_{Y}^{\prime} \Delta(Y) e^{g_{p}(\partial-Y)+g_{q}(\partial+Y)} e^{-(\Delta f) \Delta(Y)} \prod_{i} \tilde{K}\left(W_{i}\right)
\end{aligned}
$$

and

$$
\tilde{B}_{2}=\sum_{Y}^{\prime} e^{-(\Delta f) \Delta(Y)} \frac{d}{d \mu_{i}}\left(\prod_{k} K\left(W_{k}\right)\right) .
$$


Using again the bound $|\Delta(Y)| \leqq L$ and the fact that the activities $\tilde{K}$ are positive, we estimate

$$
\left|\tilde{B}_{1}\right| \leqq L \tilde{Z}(L)
$$

On the other hand

$$
\begin{aligned}
\left|\tilde{B}_{2}-\frac{d}{d \mu_{i}} Z(L)\right| & \leqq\left|e^{L|\Delta f|}-1\right| \sum_{Y}^{\prime}\left|\frac{d}{d \mu_{i}}\left(\prod_{k} K\left(W_{k}\right)\right)\right| \\
& \leqq L|\Delta f| e^{L|\Delta f|} \sum_{Y}^{\prime} \prod_{k} e^{-\left(W_{k}-\pi\left(W_{k}\right)\right)(\tau / 8-O(1))} \\
& \leqq L|\Delta f| e^{L\left(|\Delta f|+O\left(e^{-\tau / 8}\right)\right)}
\end{aligned}
$$

where we used the fact that $K$ obeys the bounds of Lemma 5.6. This completes the proof of $(5.25 \mathrm{~b})$.

Following [BF] we now implement the constraint on the heights $h_{1}, \ldots, h_{n}$ of $W_{1}, \ldots, W_{n}$ by a Fourier transformation of $\delta\left(\sum h_{i}\right)$ :

$$
\begin{gathered}
Z(L)=\frac{1}{2 \pi} \int_{-\pi}^{\pi} Z(L, p) d p, \\
Z(L, p)=\sum_{\left\{\left[W_{1}\right], \ldots,\left[W_{n}\right]\right\}} \prod_{j} K\left(W_{j}\right) e^{i h_{j} p},
\end{gathered}
$$

and similarly for $Z^{\prime}(L, p)$. If (5.21) is true, $Z(L, p)=Z^{\prime}(L, p)$ and

$$
h(L, p)=-\frac{1}{L} \log Z(L, p)
$$

can be analyzed by a convergent cluster expansion. We conclude that

$$
\begin{gathered}
\left|\frac{d^{k}}{d \mu^{k}} h(L, p)\right| \leqq O\left(e^{-b_{1} \tau}\right), \\
\left|\frac{d^{k}}{d \mu^{k}}(h(L, p)-h(p))\right| \leqq O\left(e^{-b_{1} \tau L}\right),
\end{gathered}
$$

where

$$
h(p)=-\lim _{L \rightarrow \infty} \frac{1}{L} \log Z^{\prime}(L, p)
$$

$h(L, p)$ and $h(p)$ may be rewritten as

$$
h(L, p)=\sum_{n=-\infty}^{\infty} a_{n}(L) e^{i n p},
$$

and

$$
h(p)=\sum_{n=-\infty}^{\infty} a_{n} e^{i n p},
$$

where $a_{n}(L)$ and $a_{n}$ are linear combinations of terms of the form $\prod_{i} K\left(W_{i}\right)$ with 
$\sum h_{i}=h$. Each $a_{n}$ is real, obeys a bound

$$
\left|a_{n}\right| \leqq e^{-\tau b_{1}|n|}
$$

and (by the reflection symmetry Assumption 5.5(i)), satisfies the relation $a_{n}=a_{-n}$. We conclude that $h(L, p)$ is real, which implies that

$$
Z(L, p) \geqq 0,
$$

and that

$$
h(p)=\sum_{n=-\infty}^{\infty} a_{n} \cos n p
$$

Let now

$$
h(\mu)=\inf _{p} h(p) .
$$

Since $h(p)$ is real analytic, either $h(p)$ does not depend on $p$ and

$$
Z(L)=e^{-L h(\mu)}\left(1+O\left(e^{-b_{1} \tau L}\right)\right)
$$

or

$$
h(p)>\inf h(\tilde{p}) \equiv h(\mu),
$$

except for a finite number of points $p_{1}, \ldots, p_{k}$. We can estimate the contributions of these minima to the integral (5.26a) by a saddle point analysis. It follows that there are constants $C_{k}>0$ not depending on $L$ such that

$$
Z(L)=e^{-L h(\mu)} \sum_{i=1}^{k}\left(C_{i} L^{-1 / k_{i}}+O\left(L^{-3 / k_{i}}\right)\right)
$$

as $L \rightarrow \infty$. Here $\dot{k}_{i}$ is the order of the first nonzero derivative of $h(p)$ at $p=p_{i}$. Note that $h^{\left(k_{i}\right)}\left(p_{i}\right)>0$ since $p_{i}$ is a minimum; therefore, the leading saddle point approximation

$$
\frac{1}{2 \pi} \int_{-\infty}^{\infty} \exp \left\{-L \frac{h^{\left(k_{i}\right)}}{k_{i} !}\left(p-p_{i}\right)^{k_{i}}\right\} d p=C_{i} L^{1 / k_{i}}
$$

is finite. Combining (5.32) with (5.23) and the estimates (5.20), (5.25a), and

$$
|h(\mu)| \leqq O\left(e^{-b_{1} \tau}\right),
$$

we obtain Proposition 5.2(i) with

$$
\sigma_{p q}=\left[h+\tilde{\sigma}_{p q}+\frac{1}{2}\left(e_{p}+e_{q}-f_{p}-f_{q}\right)\right] .
$$

To prove (ii) we note that the bounds (5.28a) and (5.30) imply that

$$
e^{-L O\left(e^{-\tau b_{1}}\right)} \leqq Z(L) \leqq e^{L O\left(e^{-\tau b_{1}}\right)},
$$

provided $a(\mu) L \leqq 7 \tau / 8$. Combined with (5.20) and (5.25a) we obtain Proposition 5.2(ii).

To prove (iii) we combine (5.28a), (5.30) and (5.25a) to bound

$$
\left|\frac{d}{d \mu_{i}} Z(L)\right| \leqq L O\left(e^{-b_{1} \tau}\right) Z(L) \leqq L O\left(e^{-b_{1} \tau}\right) \tilde{Z}(L),
$$


where we used the fact that $L|\Delta f| \leqq L a(\mu) \leqq O(1)$. Using (5.25b) together with (5.34) and (5.25a) we conclude that

$$
\left|\frac{d}{d \mu_{i}} \tilde{Z}(L)\right| \leqq O(L) \tilde{Z}(L)\left\{1+|\Delta f| e^{L O\left(e^{-b_{1} \tau}\right)}\right\} .
$$

Combined with the bound (5.20) this completes the proof of (iii).

We are left with the proof of (iv). We want to prove that the second derivative, $h^{(2)}(\tilde{p})>0$ whenever

$$
h(\tilde{p})=\min _{p} h(p)
$$

because this statement, together with the bound (5.32b), implies the bound (5.5). We proceed by contradiction and assume that $h^{(2)}(\tilde{p})=0$. As a consequence

$$
\begin{aligned}
h(L, \tilde{p}) & \leqq \min _{p} h(L, p)+e^{-b_{1} \tau L}, \\
\left|h^{(1)}(L, \tilde{p})\right| & \leqq e^{-b_{1} \tau L}, \quad \text { and } \\
\left|h^{(2)}(L, \tilde{p})\right| & \leqq e^{-b_{1} \tau L},
\end{aligned}
$$

for some $b_{1}>0$ and hence

$$
\begin{aligned}
Z(L, \tilde{p}) & \geqq \max _{p} Z(L, p)\left(1-e^{-\left(b_{1} \tau-O(1)\right) L}\right) \\
& \geqq \max _{p} Z(L, p)-e^{-\left(b_{1} \tau-O(1)\right) L},
\end{aligned}
$$

while

$$
\begin{aligned}
\left|Z^{(2)}(L, \tilde{p})\right| & \leqq Z(L, \tilde{p}) e^{-\left(b_{1} \tau-O(1)\right) L} \\
& \leqq e^{-\left(b_{1} \tau-O(1)\right) L} .
\end{aligned}
$$

We want to show that (5.36) and (5.37) are in contradiction with Assumption 5.5. To this end we rewrite

$$
Z(L)=\sum_{\left\{\left[W_{1}\right], \ldots,\left[W_{n}\right]\right\}_{0}} e^{g(Y)} \prod_{i} \tilde{\rho}\left(W_{i}\right),
$$

where $Y$ is a kink corresponding to $\left[W_{1}\right], \ldots,\left[W_{n}\right]$ and $g(Y)$ is a shorthand for

$$
g(Y)=g_{p}\left(\partial_{-} Y\right)+g_{q}\left(\partial_{+} Y\right)
$$

We recall that the sum goes over walls $W_{1}, \ldots, W_{n}$ such that their heights add up to zero and that $g(Y)$ may be written in the form

$$
g(Y)=\sum_{X \cap Y \neq \varnothing} k(X)=\sum_{I \subset A} \sum_{\substack{X \cap Y \neq \varnothing \\ \pi(X)=I}} k(X),
$$

where $k(X)$ depends only on that part of $Y$ which projects onto $I=\pi(X)$, is zero if $X$ is not connected and obeys a bound

$$
|k(X)| \leqq e^{-(\tau-O(1))|X|}
$$

if $a(\mu) \leqq O(1)$. 
We then approximate $Z(L)$ by

$$
Z_{\mathrm{res}}(L)=\sum_{\left\{\left[W_{1}\right], \ldots,\left[W_{n}\right]\right\}_{0}}^{\text {res }} e^{g_{\mathrm{res}}(Y)} \prod_{i} \tilde{\rho}\left(W_{i}\right),
$$

where the sums in (5.38) and (5.39) are restricted to walls $W$ and connected sets $X$ satisfying the bounds

$$
|\pi(W)|<L / 3, \quad|\pi(X)|<L / 3 .
$$

This introduces an error

$$
\left|Z(L)-Z_{\text {res }}(L)\right| \leqq e^{-b \tau L},
$$

where $b>0$ is a constant.

In the next step we introduce a partition function

$$
Z_{h}(L)=\sum_{\left\{\left[W_{1}\right], \ldots,\left[W_{n}\right]\right\}_{h}}^{\text {res }} e^{g_{\mathrm{res}}\left(W_{1}, \ldots, W_{n}\right)} \prod_{i} \tilde{\rho}\left(W_{i}\right),
$$

where the heights of $W_{1}, \ldots, W_{n}$ add up to $h \in \mathbb{Z} . g_{\text {res }}$ is defined as

$$
g_{\text {res }}\left(W_{1}, \ldots, W_{n}\right)=g_{\text {res }}(\tilde{Y})=\sum_{\substack{I \subset A \\|I|<L / 3}} \sum_{\substack{x \cap \tilde{Y} \neq \varnothing \\ \pi(X)=I}} k(X),
$$

where the "open contour" $\tilde{Y}$ corresponding to $W_{1}, \ldots, W_{n}$ is defined as follows: Let $\bar{I}$ be the connected component of $I \cup \pi\left(W_{1}\right) \cup \cdots \cup \pi\left(W_{n}\right)$ which contains $I$. Since $\bar{I}<L$ by the restrictions on $W_{1}, \ldots, W_{n}$ and $I$, it has a left and a right endpoint. Relabel $W_{1}, \ldots, W_{n}$ in such a way that $\pi\left(W_{i}\right) \subset \bar{I}$ for $i=1, \ldots, m(m \leqq n)$ and that $\pi\left(W_{i}\right)$ is to the left of $\pi\left(W_{i+1}\right)$. Translate $W_{1}, \ldots, W_{m}$ in the vertical direction so that the left boundary cube of $W_{i}$ has the same height as the right boundary cube of $W_{i+1}, i=2, \ldots, m . \tilde{Y}$ is then defined as the contour $\tilde{Y}$ with $\pi(\tilde{Y})$ which is obtained by filling the holes to the left of $W_{1}$, to the right of $W_{m}$, and between $W_{i}$ and $W_{i+1}$, $i=1, \ldots, m-1$ by flat pieces. With this definition

$$
Z_{0}(L)=Z_{\text {res }}(L)=Z(L)+O\left(e^{-b \tau L}\right)
$$

and

$$
\begin{aligned}
Z(L, p) & =\sum_{n=-\infty}^{\infty} e^{i n p} Z_{n}(L)+O\left(e^{-b \tau L}\right) \\
& =\sum_{n=-\infty}^{\infty} Z_{n}(L) \cos n p+O\left(e^{-b \tau L}\right)
\end{aligned}
$$

where we used the reflection symmetry of the model in the last step.

As a consequence of (5.43a)

$$
\max _{p} Z(L, p)=Z(L, 0)+O\left(e^{-b \tau L}\right)
$$

combined with the bound (5.36) we conclude that

$$
\sum_{n=-\infty}^{\infty} Z_{n}(L)(1-\cos n \tilde{p}) \leqq O\left(e^{-b \tau L}\right) .
$$


We now claim that

$$
Z_{n}(L)=0 \quad \text { if } \quad \cos n \tilde{p} \neq 1 .
$$

Assume that $Z_{n}(L) \neq 0$. Then there are walls $W_{1}, \ldots, W_{m}$, such that their height adds up to $n$; and such that $\tilde{\rho}\left(W_{1}\right) \neq 0, \ldots, \tilde{\rho}\left(W_{m}\right) \neq 0$. Since $Z_{n}(L)$ is a sum of positive terms

$$
Z_{n}(L) \geqq \prod_{i=1}^{m} \tilde{\rho}\left(W_{i}\right) e^{g_{\mathrm{res}}\left(W_{1}, \ldots, W_{m}\right)} .
$$

But the right-hand side of (5.46) is independent of $L$ and positive, which is a contradiction with the fact that

$$
Z_{n}(L) \leqq(1-\cos n \tilde{p})^{-1} O\left(e^{-b \tau L}\right) .
$$

This proves (5.45).

Combining (5.37), (5.45) and the bound

$$
Z^{(2)}(L, p)=\sum_{n=-\infty}^{\infty} Z_{n}(L) n^{2} \cos n p+O\left(e^{-b \tau L}\right),
$$

we finally bound

$$
\begin{aligned}
\sum_{n=-\infty}^{\infty} n^{2} Z_{n}(L) & =\sum_{n=-\infty}^{\infty} n^{2} Z_{n}(L) \cos n \tilde{p} \\
& \leqq O\left(e^{-b \tau L}\right),
\end{aligned}
$$

from which we conclude that

$$
Z_{n}(L) \leqq O\left(e^{-b \tau L}\right),
$$

for all $n \neq 0$. As a consequence

$$
\tilde{\rho}(W) e^{g_{\mathrm{res}}(W)} \leqq O\left(e^{-b \tau L}\right),
$$

for all small walls $W$ with height $h \neq 0$ (use that fact that (5.42) is a sum of positive terms). The left-hand side of (5.47) is independent of $L$. Therefore, $\tilde{\rho}(W)=0$ for all small walls with height $h \neq 0$, which is in contradiction with Assumption 5.5(iii).

Remark. The above proof shows that Assumption 5.5(iii) is a necessary and sufficient condition for the bound (5.5): Either $\tilde{\rho}(W) \neq 0$ for at least one wall of height $h \neq 0$ and (5.5) is valid, or $\tilde{\rho}(W)=0$ for all small walls of height $h \neq 0$, and

$$
Z(L)=Z(L, p=0)=e^{-h L}\left(1+O\left(e^{-b \tau L}\right)\right),
$$

from which we conclude that

$$
\Gamma_{p q}^{(1)}=e^{-\left(f+\sigma_{p q}\right) L}\left(1+O\left(e^{-b \tau L}\right)\right) .
$$

We finally prove Theorem 2.4, using Propositions 5.1 through 5.3.

Proof of Theorem 2.4. By Propositions 5.1 and 5.2,

$$
e^{-\left(f+\tilde{\sigma}_{p q}\right) L^{v}} \leqq e^{-\left(\left(f_{p}+f_{q}\right) / 2+\sigma_{p q}-O(1)\right) L^{v}} \leqq \Gamma_{p q}^{(1)}(L) e^{O(1) L^{v}}
$$


provided $a(\mu) L^{\nu} \leqq O(1)$. As a consequence of this bound and (5.6b),

$$
\Gamma_{p q}^{(1)}(L) \leqq \Gamma_{p q}(L)\left(1+e^{O(1) L^{v}} e^{-\tau\left(L^{v}-1\right)}\right) .
$$

Combining these bounds with (5.6a), we get the two bounds of Theorem 2.4 (i); combining them with (5.6b), we get the bound (2.27) for $v=1$. For $v \geqq 2$,

$$
\left|\frac{d^{k}}{d \mu^{k}} \Gamma_{p q}^{(1)}(L)\right| \leqq \Gamma_{p q}^{(1)}(L) O\left(L^{v k}\right)
$$

by Proposition 5.1 and the fact that derivatives of $\tilde{\sigma}_{p q}+\frac{f_{p}+f_{q}}{2}$ are bounded by $O(1)$. Combined with (5.6b), (5.48) and (5.49) we get Theorem 2.4 (iii), while (5.4), (5.48) and (5.49) imply Theorem 2.4 (iv) for $v=1$. For $v \geqq 2$ the bound (2.27) follows from $(2.28)$.

\section{Appendix A}

In this appendix we summarize the main ideas of the construction of the truncated free energies $f_{q}$ as introduced in Sect. 2 of [BK]. This construction uses volumes $W$ which are part of $\mathbb{R}^{v+1}$ rather than $A \times T$, so we will only consider contours $Y$ which may be considered as contours $Y$ in $\mathbb{R}^{v+1}$ in this appendix.

For a contour $(Y, q(\cdot))$ in $\mathbb{R}^{v+1}$, we define Ext $Y$ as the finite component of $Y^{c}=\mathbb{R}^{d+1} \backslash Y$, Int ${ }_{m} Y$ as the union of all finite components $C$ of $Y^{c}$ for which $q(\cdot)=m$ on $\partial C$, Int $Y$ as the union $\bigcup_{m} \operatorname{Int}_{m} Y$ and $V(Y)$ as $U \cup$ Int $Y$. We say that $(Y, q(\cdot))$ is a $q$-contour if $q(\cdot)=q$ on $\partial \operatorname{Ext} Y$, and say that $Y_{\bar{\alpha}}$ is an exterior countour of a collection $\left\{Y_{\alpha}\right\}$ of nonoverlapping contours if $Y_{\bar{\alpha}} \cap \operatorname{Int} Y_{\alpha}=0$ for all $\alpha \neq \bar{\alpha}$. Finally, a contour $Y$ is called a contour in $W \subset \mathbb{R}^{v+1}$, if $V(Y) \subset W$.

Given these notions we introduce the partition function $Z_{q}(W)$ with boundary condition $q$ as

$$
Z_{q}(W)=\sum_{\left\{Y_{x}\right\}} \prod_{\alpha} \rho\left(Y_{\alpha}\right) \prod_{m} e^{-e_{m}\left|R_{m}\right|},
$$

where the sum goes again (cf. (2.3) in Sect. 2) over collections of nonoverlapping contours with compatible boundary labels, with the additional restrictions that the external contours of $\left\{Y_{a}\right\}$ are $q$-contours, and that all contours in $\left\{Y_{a}\right\}$ are contours in $W$. As before, $R_{m}$ is the union of all connected components of $W \backslash \bigcup_{\alpha} Y_{\alpha}$
which have boundary condition $m$.

For many purposes we need a different expression for $Z_{q}(W)$ which eliminates the compatibility of boundary conditions on contours. Fixing the external contours in the representation (A.1) for $Z_{q}(W)$ and resumming all others we obtain a factor $\prod_{m} Z_{m}\left(\operatorname{Int}_{m} Y^{q}\right)$, for each external contour. This yields the expression

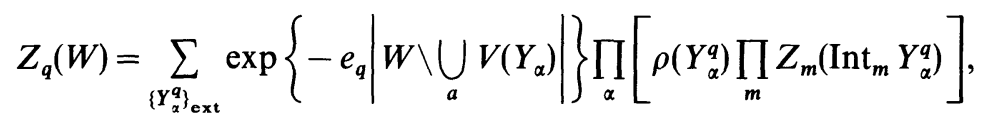

where the sum goes over the sets $\left\{Y_{\alpha}^{q}\right\}$ of mutually external $q$-contours (i.e., $V\left(Y_{\alpha}\right)=Y_{\alpha} \cup$ Int $Y_{\alpha}$ and $V\left(Y_{\bar{\alpha}}\right)=Y_{\bar{\alpha}} \cup$ Int $Y_{\bar{\alpha}}$ have no common cubes for $\left.\alpha \neq \bar{\alpha}\right)$. We 
divide each $Z_{m}$ by the corresponding $Z_{q}$ and multiply it back again. Iterating this procedure we get

$$
Z_{q}(W)=e^{-e_{q}|W|} \sum_{\left\{Y_{\alpha}^{q_{q}}\right\}} \prod_{\alpha} K\left(Y_{\alpha}^{q}\right),
$$

where the sum goes over sets $\left\{Y_{\alpha}^{q}\right\}$ of $q$-contours such that $Y_{\alpha}^{q}$ and $Y_{\bar{\alpha}}^{q}$ have no common cubes for $\alpha \neq \bar{\alpha}$, and $K\left(Y^{q}\right)$ is defined by

$$
K\left(Y^{q}\right)=\rho\left(Y^{q}\right) e^{e_{q}\left|Y^{q}\right|} \prod_{m} \frac{Z_{m}\left(\operatorname{Int}_{m} Y^{q}\right)}{Z_{q}\left(\operatorname{Int}_{m} Y^{q}\right)} .
$$

Note that (A.3) is purely formal at this point since $Z_{q}\left(\operatorname{Int}_{m} Y^{q}\right)$ might be zero for some contours $Y^{q}$ (we have introduced the superscript $q$ to emphasize the fact that $Y$ is a $q$-contour).

The truncated free energy $f_{q}$ is then introduced as the free energy of a modified model

$$
f_{q}=-\lim _{W \rightarrow \mathbb{Z}^{+1}} \frac{1}{|W|} \log Z_{q}^{\prime}(W)
$$

where

$$
Z_{q}^{\prime}(W)=e^{e_{q}|W|} \sum_{\left\{Y_{\alpha}^{q}\right\}} \prod_{\alpha} K^{\prime}\left(Y_{\alpha}^{q}\right)
$$

and $K^{\prime}(Y)$ is defined inductively as follows.

Assume that $K^{\prime}(Y)$ has already been defined for all contours $Y$ with diam $Y<n$, $n \in \mathbb{N}$, and that it obeys a bound

$$
\left|K^{\prime}(Y)\right| \leqq \varepsilon^{|Y|},
$$

for some small $\varepsilon$. Then $Z_{q}^{\prime}(W)$ is defined for all $q$ and all volumes $W$ with diam $W<n$. Its logarithm can be controlled by a convergent cluster expansion and $Z_{q}^{\prime}(W) \neq 0$. For $q$-contours of diameter $n, K^{\prime}\left(Y^{q}\right)$ is then defined by

$$
\begin{aligned}
K^{\prime}\left(Y^{q}\right) & =\left[\rho\left(Y^{q}\right) e^{e_{q}\left|Y^{q}\right|} \prod_{m} \frac{Z_{m}\left(\operatorname{Int}_{m} Y^{q}\right)}{Z_{q}^{\prime}\left(\operatorname{Int}_{m} Y^{q}\right)}\right] \chi^{\prime}\left(Y^{q}\right), \\
\chi^{\prime}\left(Y^{q}\right) & =\prod_{m} \chi\left(\log \left|Z_{q}^{\prime}\left(V\left(Y^{q}\right)\right)\right|-\log \left|Z_{m}^{\prime}\left(V\left(Y^{q}\right)\right)\right|+\alpha\left|Y^{q}\right|\right),
\end{aligned}
$$

where $\alpha$ may be chosen arbitrarily in a range $O(1) \leqq \alpha \leqq \tau-O(1)$, and $0 \leqq \chi \leqq 1$ is a smoothed characteristic function obeying the conditions $\chi(x)=0$ if $x \leqq-1$, $\chi(x)=1$ if $x \geqq 1$, and $\left|d^{k} \chi(x) / d x^{k}\right| \leqq O(1)$ for $1 \leqq k \leqq 4$. As the final step of the construction one then has to establish the bound (A.7) for the contours of diameter $n$. Since all other partition functions in (A.8) can be controlled by convergent expansion due to the inductive assumption, the only nontrivial bound is an upper bound on $Z_{m}\left(\operatorname{Int}_{m} Y^{q}\right)$. This is done by a technique first introduced by Zahradnik in $[\mathrm{Z}]$ and carried out in detail in the appendix of [BK]. As a corollary of this proof, one obtains the following:

Lemma A.1 [BK]. Assume that $|\rho(Y)| \leqq e^{-\left(\tau+e_{0}\right)|Y|}$ for all contours $Y$. Then the following statements are true provided $\tau$ is large enough and $3 \leqq \alpha \leqq \tau-O(1)$.

(i) $K^{\prime}(Y)$ is well defined for all $Y$ and obeys a bound $\left|K^{\prime}(Y)\right| \leqq e^{-(\tau-\alpha-o(1)) \mid Y}$. 
(ii) If $a_{q} \operatorname{diam} W \leqq \alpha-2$, then $Z_{q}(\tilde{W}) \neq 0$ for all $\tilde{W} \subset W$ and $K^{\prime}\left(Y^{q}\right)=K\left(Y^{q}\right)$ for all q-contours $Y^{q}$ in $W$.

(iii) $\left|Z_{q}(W)\right| \leqq e^{-f|W|+|\partial W|}$ for all finite volumes $W \subset \mathbb{R}^{v+1}$.

Here $f=\min _{m} f_{m}$ and $a_{q}=f_{q}-f$.

Remark. A close inspection of the proof of Lemma A.1 as given in [BK] shows that the condition $a_{q}$ diam $W \leqq \alpha-2$ in statement (ii) can be replaced by $a_{q}|\pi(W)| \leqq \alpha-2$, where $\pi(\cdot)$ is the projection on a $v$-dimensional hypersurface $S$ in $\mathbb{R}^{v+1}$ orthogonal to one of the coordinate directions and $|\pi(W)|$ denotes the $v$-dimensional volume of $\pi(W)$. To prove this statement, the following changes should be made in Sect. 2 and in the appendix of [BK]:

( $\alpha$ ) The conditions $a_{q}$ diam $Y^{q} \leqq \alpha-2$ and $a_{q}$ diam $V \leqq \alpha-2$ in Lemma 2.1 should be replaced by $a_{q}\left|\pi\left(Y^{q}\right)\right| \leqq \alpha-2$ and $a_{q}|\pi(V)| \leqq \alpha-2$, respectively. Similarly, in Lemma A.1 and Lemma A.2.

( $\beta$ ) The bound $\left|V\left(Y^{q}\right)\right| \leqq\left|Y^{q}\right| \operatorname{diam} Y^{q}$ which was used in the proof of Lemma A.1 should be replaced by

$$
\left|V\left(Y^{q}\right)\right| \leqq l_{\perp}\left(Y^{q}\right)\left|\pi\left(Y^{q}\right)\right| \leqq\left|Y^{q}\right|\left|\pi\left(Y^{q}\right)\right|,
$$

where $l_{\perp}\left(Y^{q}\right)$ is the minimal distance between two surfaces $S_{1}, S_{2}$ parallel to $S$ such that $Y^{q}$ lies between $S_{1}$ and $S_{2}$.

$(\gamma)$ The definition of a small contour given in the proof of Lemma A.3 should be replaced by " $Y^{q}$ is small if $a_{q}\left|\pi\left(Y^{q}\right)\right| \leqq \alpha-2$." Note the fact that $\left|Y^{q}\right| \geqq(\alpha-2) / a_{q}$ for all larger contours, which is implicitly used in the proof of Lemma A.3, is still true with this definition.

( $\delta$ ) Even though not necessary, it is convenient to replace the induction on the diameter $Y$ by an induction on $|V(Y)|$.

Remark. The bounds of Lemma A.1 can be generalized to derivatives (see [BK]). One obtains (for $|k| \leqq 4$ )

$$
\left|\frac{d^{k}}{d \mu^{k}} Z_{q}(W)\right| \leqq \text { const }|W|^{|h|} e^{-f|W|+|\partial W|}
$$

and

$$
\left|\frac{d^{k}}{d \mu^{k}} K^{\prime}\left(Y^{q}\right)\right| \leqq e^{-(\tau-\alpha-O(1))\left|Y^{q}\right|} .
$$

Using these bounds and the fact that $K^{\prime}\left(Y^{q}\right)=K\left(Y^{q}\right)$ if $a_{q}\left|\pi\left(Y^{q}\right)\right| \leqq \alpha-2$, we will now show that

$$
\begin{aligned}
\left|\frac{d^{k}}{d \mu^{k}} K\left(Y^{q}\right)\right| & \leqq e^{a_{q}\left|\ln t Y^{q}\right|-(\tau-O(1))\left|Y^{q}\right|} \\
& \leqq e^{-\left(\tau-a_{q}\left|\pi\left(Y^{q}\right)\right|-O(1)\right)\left|Y^{q}\right|}
\end{aligned}
$$

if $a_{q}\left|\pi\left(Y^{q}\right)\right| \leqq \alpha-2$. 
Proof. Due to the bound (A.9) and the fact that $\mid$ Int $\left.Y\right|^{|k|} \leqq e^{o(1)|Y|}$, we have

$$
\left|\frac{d^{k}}{d \mu^{k}} \prod_{m} Z_{m}\left(\operatorname{Int}_{m} Y^{q}\right)\right| \leqq e^{-f\left|\operatorname{Int} Y^{q}\right|+O(1)\left|Y^{q}\right|},
$$

for each $q$-contour $Y^{q}$. If $\left|\pi\left(Y^{q}\right)\right| a_{q} \leqq \alpha-2, \log Z_{q}\left(\operatorname{Int}_{m} Y^{q}\right)=\log Z_{q}^{\prime}\left(\operatorname{Int}_{m} Y^{q}\right)$ can be analyzed by a convergent cluster expansion and

$$
\left|\frac{d^{k}}{d \mu^{k}} \prod_{m} Z_{q}^{-1}\left(\operatorname{Int}_{m} Y^{q}\right)\right| \leqq e^{f_{q}\left|\operatorname{Int} Y^{q}\right|+O(1)\left|Y^{q}\right|},
$$

due to the bound (A.10). Combined with the a priori bounds on $\rho$ and the fact that $\mid$ Int $Y^{q}|\leqq| Y^{q}|| \pi\left(Y^{q}\right) \mid$, we obtain (A.11).

Up to now, we have not specified a choice of $\alpha$. Since both the bound (A.9) as well as the bound (A.11) do not involve $\alpha$ explicitly, while (A.11) has a wider range of validity if $\alpha$ is chosen larger, it is favorable to choose $\alpha$ large (but one has to respect the restriction $\alpha \leqq \tau-O(1))$. We chose $\alpha$ as

$$
\alpha=2+\frac{7}{8} \tau \text {. }
$$

With this choice of $\alpha$, we summarize the main results of this section in the following:

Lemma A.2. The following statements hold provided $\tau$ is chosen large enough:

(i) For all $q$, all volumes $W \subset \mathbb{R}^{v+1}$ and all $|k| \leqq 4$,

$$
\left|\frac{d^{k}}{d \mu^{k}} Z_{q}(W)\right| \leqq \text { const }|W|^{|k|} e^{-f|W|+|\partial W|} .
$$

(ii) Assume that $|k| \leqq 4$ and that $a_{q}\left|\pi\left(Y^{q}\right)\right| \leqq \frac{7}{8} \tau$. Then,

$$
\begin{aligned}
\left|\frac{d^{k}}{d \mu^{k}} K\left(Y^{q}\right)\right| & \leqq e^{a_{q}\left|\ln t Y^{q}\right|-(\tau-O(1))\left|Y^{q}\right|} \\
& \leqq e^{-\left[\tau-a_{q}\left|\pi\left(Y^{q}\right)\right|-O(1)\right]|Y q|} .
\end{aligned}
$$

(iii) If $a_{q}|\pi(W)| \leqq \frac{7}{8} \tau$, then $Z_{q}(\tilde{W}) \neq 0$ for all $\tilde{W} \subset W$ and $Z_{q}(W)=e^{-e_{q}|W|} \sum_{\left\{Y_{\alpha}^{q}\right\}} \prod_{\alpha} K\left(Y_{\alpha}^{q}\right)$.

Remark. The condition

$$
\left|\pi\left(Y^{q}\right)\right| a_{q} \leqq \frac{7}{8} \tau
$$

is more convenient for the applications in Sect. 3 than the original condition

$$
|\operatorname{diam} Y| a_{q} \leqq \frac{7}{8} \tau,
$$

because the bound $|V(Y)| \leqq|\pi(Y)||Y|$ remains true for certain topologically nontrivial contours (called ordinary contours in Sect. 3), while the bound $|V(Y)| \leqq|\pi(Y)|$ diam $Y$ becomes false in this context. This is the reason why we state the generalization of Lemma A. 2 in Sect. 3 using the condition $|\pi(Y)| a_{q} \leqq 7 \tau / 8$. 


\section{References}

[BF] Bricmont, J., Fröhlich, J.: Statistical mechanical methods in particle structure analysis of lattice field theories II: Scalar and surface models. Commun. Math. Phys. 98, 553-578 (1985)

[BI1] Borgs, C., Imbrie, J. Z.: A unified approach to phase diagrams in field theory and statistical mechanics. Commun. Math. Phys. 123, 305-328 (1989)

[BI2] Borgs, C., Imbrie, J. Z.: Crossover finite-size scaling at first-order transitions, preprint (1992)

[BK] Borgs, C., Kotecký, R.: Finite-size effects at asymmetric first-order phase transitions, preprint. A rigorous theory of finite-size scaling at first order phase transitions. J. Stat. Phys. 61, 79 (1990)

[BFW] Borgs, C., Fröhlich, J., Waxler, R.: The phase structure of the large $N$ lattice Higgs model. Nucl. Phys. B328, 611-638 (1989)

[BKM] Borgs, C., Kotechý, R., Miracle-Solé, S.: Finite-size scaling for Potts models. J. Stat. Phys. 62, 529 (1991)

[BZ] Brézin, E., Zinn-Justin, J.: Finite size effects in phase transitions. Nucl. Phys. B257, 867-893 (1985)

[CJBZ] Cabrera, G. G., Jullien, R., Brézin, E., Zinn-Justin, J.: Test of finite-size scaling in first order phase transitions. J. Phys. 47, 1305-1313 (1986)

[D] Dobrushin, R. L.: Gibbs states describing the coexistence of phases for a three-dimensional Ising model. Theor. Prob. Appl. 17, 582-600 (1972). Investigation of Gibbsian states for three-dimensional lattice systems. Theor. Prob. Appl. 18, 253-271 (1973)

[FB] Fisher, M. E., Berker, A. N.: Scaling for first-order phase transitions in thermodynamic and finite systems. Phys. Rev. B26, 2507-2513 (1982)

[G] Gallavotti, G.: The phase separation line in the two-dimensional Ising model. Commun. Math. Phys. 27, 103-136 (1972)

[HKZ] Holichy, P., Kotecký, R., Zahradnik, M.: Rigid interfaces for lattice models at low temperatures. J. Stat. Phys. 50, 755-812 (1988)

[I] Isakov, S. N.: Nonanalytic features of the first order phase transition in the Ising model. Commun. Math. Phys. 95, 427-433 (1984)

[IJW] Imbrie, J. Z., Janowsky, S. A., Weitsman, J.: Space dependent Dirac operators and effective quantum field theory for fermions. Commun. Math. Phys. 135, 421-442 (1991)

[J] Janowsky, S. A.: The phase structure of the two-dimensional $N=2$ Wess-Zumino model. Harvard University thesis (1990)

[JLW] Jaffe, A., Lesniewski, A., Weitsman, J.: Index of a family of Dirac operators on loop space. Commun. Math. Phys. 112, 75-88 (1987); The loop space $S^{1} \rightarrow \mathbb{R}$ and supersymmetric quantum fields. Ann. Phys. 183, 337-351 (1988); The two-dimensional, $N=2$ Wess-Zumino models on a cylinder. Commun. Math. Phys. 114, 147-165 (1988)

[JW1] Janowsky, S. A., Weitsman, J.: The phase structure of the two-dimensional $N=2$ Wess-Zumino model. Commun. Math. Phys. 142, 25-66 (1991)

[JW2] Janowsky, S. A., Weitsman, J.: A vanishing theorem for supersymmetric quantum field theory and finite-size effects in multiphase cluster expansions. Commun. Math. Phys. 143, 85-97 (1991)

[LMMRS] Lanait, L., Messager, A., Miracle-Solé, S. Ruiz, J., Shlossman, S.: Interfaces in the Potts Model I: Pirogov-Sinai theory of the Fortuin-Kastelyn representation. Commun. Math. Phys. 140, 81-92 (1991)

[P] Privman, V. (ed.): Finite-size scaling and numerical simulation of statistical systems. Singapore: World Scientific 1990

[PF] Privman, V., Fisher, M. E.: Finite-size effects at first-order transitions. J. Stat. Phys. 33, 385-417 (1983) 